\title{
Efficient Local Search based on Dynamic Connectivity Maintenance for Minimum Connected Dominating Set
}

\author{
Xindi Zhang \\ Bohan Li \\ Shaowei Cai \\ State Key Laboratory of Computer Science \\ Institute of Software, Chinese Academy of Sciences \\ Beijing, China \\ School of Computer Science and Technology \\ University of Chinese Academy of Sciences \\ Beijing, China \\ Yiyuan Wang \\ School of Computer Science and Information Technology \\ Northeast Normal University, China \\ Key Laboratory of Applied Statistics of MOE \\ Northeast Normal University, China
}

\author{
ZHANGXD@IOS.AC.CN \\ LIBOHAN19@MAILS.UCAS.AC.CN \\ SHAOWEICAI.CS@GMAIL.COM
}

YIYUANWANGJLU@126.COM

\begin{abstract}
The minimum connected dominating set (MCDS) problem is an important extension of the minimum dominating set problem, with wide applications, especially in wireless networks. Most previous works focused on solving MCDS problem in graphs with relatively small size, mainly due to the complexity of maintaining connectivity. This paper explores techniques for solving MCDS problem in massive real-world graphs with wide practical importance. Firstly, we propose a local greedy construction method with reasoning rule called 1hopReason. Secondly and most importantly, a hybrid dynamic connectivity maintenance method (HDC+) is designed to switch alternately between a novel fast connectivity maintenance method based on spanning tree and its previous counterpart. Thirdly, we adopt a two-level vertex selection heuristic with a newly proposed scoring function called chronosafety to make the algorithm more considerate when selecting vertices. We design a new local search algorithm called FastCDS based on the three ideas. Experiments show that FastCDS significantly outperforms five state-of-the-art MCDS algorithms on both massive graphs and classic benchmarks.
\end{abstract}

\section{Introduction}

With a series of computational challenges brought by the rapid increase of massive data in recent years, most existing algorithms become ineffective when solving NP-hard problems on massive data sets. Thus, in the last decade, many researchers devoted their efforts to developing new algorithms to deal with massive real-world graphs. In this paper, we consider an interesting graph theory problem, namely the minimum connected dominating set (MCDS) problem, and propose a series of effective techniques focusing on solving MCDS problem in massive graphs. 
Given an undirected connected graph $G=(V, E)$, a set $D \subseteq V$ is called a dominating set if each vertex in $V$ either belongs to $D$ or is adjacent to at least one vertex in $D$. The minimum dominating set (MDS) problem is to find a dominating set with the minimum number of vertices in the given graph. An important generalization of MDS is the MCDS problem, whose goal is to find a MDS that forms a connected subgraph in the given graph. An important application of MCDS problem is to generate a virtual backbone in wireless networks (Yu et al., 2013) such as mobile ad hoc networks (Al-Karaki \& Kamal, 2008), wireless sensors networks (Misra \& Mandal, 2009) and vehicular ad hoc networks (Chinnasamy et al., 2019). Specifically, MCDS problem plays a significant role in broadcast routing (Cheng et al., 2006; Ni et al., 1999; Wu \& Dai, 2003), power managing (Chen et al., 2002; Deb et al., 2003) and fiber optical networks (Chen et al., 2010; Sen et al., 2008). In addition, MCDS problem has also lots of applications in many other fields, such as system biology (Milenković et al., 2011) and aviation network (Li et al., 2020b). Moreover, MCDS problem is equivalent to the maximum leaf spanning tree problem (Lucena et al., 2010; Fernau et al., 2011; Binkele-Raible \& Fernau, 2012; Solis-Oba et al., 2017).

\subsection{Previous Works}

It is well known that MCDS problem is NP-hard (Kann, 1992). Several exact algorithms (Fomin et al., 2008; Simonetti et al., 2011; Fan \& Watson, 2012; Gendron et al., 2014) and approximation algorithms (Cheng et al., 2003; Ruan et al., 2004; Khuller \& Yang, 2019) have been designed for MCDS problem. For example, the improved 1-hop and 2-hop local information greedy algorithms were proposed in (Khuller \& Yang, 2019), with approximation ratio of $H\left(\Delta_{G}\right)+2 \sqrt{H\left(\Delta_{G}\right)}+1$ and $H\left(2 \Delta_{G}+1\right)+1$ respectively, where $H$ is the harmonic function and $\Delta_{G}$ is the maximum degree in the graph. Nevertheless, these algorithms are either too time-consuming or have poor performance in practice, especially in the context of massive graphs.

Because of its NP-hardness, much of the research effort in the past decade concerned with solving MCDS problem has focused on heuristic algorithm aiming to obtain a good solution within a reasonable time. Two algorithms called MCDS/SA and MCDS/TS based on simulated annealing and tabu search were proposed (Morgan \& Grout, 2007). Hedar and Ismail designed a simulated annealing algorithm with stochastic local search for MCDS problem (Hedar \& Ismail, 2012). Later, Jovanovic and Tuba designed an ant colony optimization algorithm with a so-called pheromone correction strategy (Jovanovic \& Tuba, 2013). A greedy random adaptive search procedure that incorporated a local search procedure based on a greedy function and tabu search was described (Li et al., 2017). Wu et al. used a restricted swap-based neighborhood to improve the tabu search procedure, resulting in the RNS-TS algorithm (Wu et al., 2017). Two meta-heuristics based on genetic algorithms and simulated annealing were designed to solve MCDS problem (Hedar et al., 2019). Li et al. presented a multi-start local search algorithm called MSLS based on three mechanisms including a vertex score, configuration checking, and vertex flipping (Li et al., 2019). Finally, a meta-heuristic algorithm called ACO-RVNS (Bouamama et al., 2019) was proposed, based on ant colony optimization and reduced variable neighborhood search. Moreover, ACO-RVNS has also been applied to weighted version of MCDS problem. Experiments showed that, for classic graphs with fewer than 5000 vertices, RNS-TS, MSLS, 
and ACO-RVNS obtained similar state-of-the-art performance, while the performance of these algorithms was different on solving massive graphs.

\subsection{Contributions}

Previous MCDS algorithms have made progress in solving classic graphs, but these algorithms still cannot handle massive graphs with millions of vertices. In this work, we focus on solving MCDS problem on massive graphs and propose an efficient local search algorithm called FastCDS. The main technical contributions of this paper are as follows.

First, we propose a construction algorithm based on reasoning rule called 1hopReason. According to simple preprocessing, vertices are divided into three sets, including a set of vertices that are contained in each feasible solution, a set of vertices that are not contained in any optimal solution, and the third set consisting of the remaining vertices. The 1 hopReason algorithm constructs a solution according to the reasoning rule which exploits the different parts of vertices.

The second idea, which is also the most important contribution of this work, concerns with the connectivity maintenance. For combinatorial optimization problem with connectivity constraint, a key factor to the performance is the connectivity maintenance method, especially when the graphs are large. Previous connectivity maintenance methods make the algorithm explore rather large parts of the search space, but they become futile when faced with very large search space. To overcome this issue, we propose a novel tree-based connectivity maintenance (TBC for short) method, inspired by spanning trees. Compared to previous counterparts, the TBC method has very low complexity, while limiting the algorithm to explore a relatively small part of the search space. To obtain a balance between diversification and intensification, we design the a hybrid dynamic connectivity maintenance method (HDC) method to dynamically switch between previous connectivity maintenance methods and TBC during the search process. Furthermore, HDC is enhanced by reconstructing spanning tree when the algorithm falls into local minima for certain time, resulting in $\mathrm{HDC}+$ method.

Finally, we designed a two-level vertex selection heuristic. By defining a new vertex property called safety, which takes into account the difference among dominated vertices and the diversity of solution, we propose a novel vertex scoring function called chrono-safety based on different scoring functions including the safety property and history information of vertices. Comparing to previous MCDS algorithms which only use one scoring function, the proposed two-level vertex selection rule uses the chrono-safety scoring function as a supplement of traditional scoring function to make itself more considerate.

Extensive experiments are carried out to evaluate FastCDS on classic benchmarks used in previous literature and on massive graphs from real-world applications. Experimental results indicate that FastCDS outperforms five state-of-the-art MCDS heuristic algorithms on most instances, and confirm the effectiveness of our proposed strategies.

This paper is an extended and improved version of a conference paper ( $\mathrm{Li}$ et al., 2020a), where a local search algorithm for MCDS problem called NuCDS is proposed. The HDC method and the safety-based vertex selection heuristic (Li et al., 2020a) have been presented in the conference version of this article. The new contributions in this article are summarized as follows: 
- A new effective construction method called 1hopReason;

- An improved version of HDC method called HDC+;

- A two-level vertex selection method with the optimized chrono-safety scoring function;

- More experiments to evaluate the performance of our algorithm, comparing it with five state-of-the-art algorithm, including NuCDS;

- Comprehensive assessment for each strategy.

\subsection{Paper Organization}

Some preliminary knowledge is introduced in Section 2. In Section 3, we propose an efficient construction method with reasoning rule called 1hopReason. In Sections 4 and 5, we describe the two main ideas in the local search process, including the connectivity maintenance method HDC+ and the two-level vertex selection heuristic with chrono-safety as the secondary scoring function. We describe the FastCDS algorithm in Section 6. Experimental results and further analyses are presented in Section 7. Finally, we give some concluding remarks in Section 8.

\section{Preliminaries}

In this section, we introduce the basic definitions and notations that will be used in this paper, and then we briefly overview two strategies adopted in FastCDS, called two-level configuration checking and best from multiple selection.

\subsection{Basic Definitions and Notation}

An undirected graph $G=(V, E)$ consists of a vertex set $V$ and an edge set $E$. For an edge $e=\{u, v\}$, vertices $u$ and $v$ are the endpoints of the edge. The distance between two vertices $u$ and $v$, denoted by $\operatorname{dist}(u, v)$, is the number of edges in a shortest path from $u$ to $v$. For vertex $v$, its $i$ th level neighborhood is $N_{i}(v)=\{u \mid \operatorname{dist}(u, v)=i\}$, and its $i$ th level closed neighborhood is $N_{i}[v]=N_{i}(v) \cup\{v\}$. We denote $N^{k}(v)=\bigcup_{i=1}^{k} N_{i}(v)$. Also, the $i$ th level neighborhood and the closed neighborhood of a vertex set $S \subseteq V$ are defined as $N_{i}(S)=\bigcup_{v \in S} N_{i}(v) \backslash S$ and $N_{i}[S]=\bigcup_{v \in S} N_{i}[v]$, respectively. The first level neighborhood symbol $N_{1}$ is usually denoted as $N$. The degree of a vertex $v$ in $G$, denoted as $d(v)$, is defined as $|N(v)|$. For the given graph $G, \Delta_{G}$ is the corresponding maximum degree. $G[S]=\left(V_{S}, E_{S}\right)$ is a subgraph in $G$ induced by $S$ such that $V_{S}=S$ and $E_{S}$ consists of all the edges in $E$ whose endpoints are in $S$.

An undirected graph $G=(V, E)$ is connected when it has at least one vertex and there is a path between every pair of vertices. For the given graph $G$, a clique $C$ of $G$ is a subset of $V$ where each pair of vertices in $C$ is adjacent.

Definition 1 Given an undirected connected graph $G$, a vertex in $G$ is an articulation vertex iff removing it, together with the edges connected to it, disconnects the graph. The articulation vertex set of $G$ is denoted as $A(G)$. 
Given a graph $G=(V, E)$, for a vertex set $D \subseteq V$, a vertex $v \in V$ is dominated by $D$ if $v \in N[D]$, and is non-dominated otherwise. If $D$ dominates all vertices, then $D$ is a dominating set, and if its induced subgraph is also connected then it is a connected dominating set. For a given graph $G$, the aim of the minimum connected dominating set (MCDS) problem is to find the connected dominating set $D$ with the smallest size.

During the search process, we use $D \subseteq V$ to denote a candidate solution. If the algorithm only considers vertices in the $N_{i}(D)$ to be moved (i.e. added into or removed from the candidate solution), then we say that it uses $i$-hop information.

\subsection{Two-Level Configuration Checking}

Configuration checking (CC) (Cai \& Su, 2011) is a strategy aiming to handle the cycling problem in local search, i.e., revisiting a candidate solution that has been visited recently. It can be briefly described as below. The configuration of a vertex $v$ refers to the states (being selected or not) of all its neighboring vertices. If the configuration of $v$ remains the same as the last time it was removed from the candidate solution, then $v$ is forbidden to be added into the candidate solution. Typically, CC is implemented with a Boolean array ConfChange whose size equals the number of vertices in the given graph.

A variant of $\mathrm{CC}$ named two-level configuration checking $\left(\mathrm{CC}^{2}\right)$ was proposed to improve a local search algorithm for MWDS (Wang et al., 2017). In $\mathrm{CC}^{2}$, the configuration of a vertex $v$ is defined to be a vector consisting of states of vertices in $N^{2}(v)$. A vertex is considered configuration-changed, if the value of any bit of the vector has changed. The $\mathrm{CC}^{2}$ strategy forbids any vertex to be added into the candidate solution if it is not configurationchanged since its last removal from the candidate solution.

The $\mathrm{CC}^{2}$ strategy works as follows.

\section{Updating rules:}

1. At the beginning of the local search, for each vertex $v$, ConfChange $[v]$ is set to 1 ;

2. When vertex $v$ is removed (from current candidate solution), ConfChange $[v]$ is reset to 0 , and ConfChange $[u]$ is set to 1 for all $u \in N^{2}(v)$;

3. When vertex $v$ is added (into current candidate solution), ConfChange $[u]$ is set to 1 for all $u \in N^{2}(v)$.

Using rule: When choosing an added vertex $v, \mathrm{CC}^{2}$ forbids any vertex to be added into the candidate solution if its configuration has not been changed, i.e., ConfChange $[v]=0$.

\subsection{Best from Multiple Selection Heuristic}

Local search algorithms usually need to pick an element (e.g., a vertex or variable) from a candidate set. For solving large instances, a commonly used heuristic is the best from multiple selection (BMS) heuristic (Cai, 2015), which is a probabilistic sampling method.

To gain a balance between the quality of the selected element and the time complexity of selection process, BMS randomly chooses $k$ elements from a given candidate set, and then returns the best one according to a selection rule, where $k$ is a parameter. BMS has been proved with theoretical basis that it can return an excellent quality element on large scale instances. 


\section{An Effective Construction Algorithm for MCDS with Reasoning Rule}

In order to handle massive sparse graphs, it is essential to efficiently construct a highquality initial solution for local search algorithm. We propose a simple and efficient greedy algorithm based on reasoning rules for constructing a feasible solution as the initial solution of local search.

\subsection{The Reasoning Rule}

Proposition 1 Given a simple incomplete graph $G=(V, E)$, a vertex $v$ is not contained in any optimal solution of $M C D S$ if its closed neighborhood $N[v]$ forms a clique.

Proof: Let $D^{\text {opt }}$ be an arbitrary optimal solution of MCDS problem for graph $G$, and $v$ be a vertex such that its closed neighborhood $N[v]$ forms a clique $C$. Supposing that $v \in D^{o p t}$, since $D^{o p t}$ is connected and $v$ connects only to vertices in $C$, there must be at least one vertex $u \in C \backslash\{v\}$ in $D^{o p t}$. Since $v$ is dominated by $u, D^{o p t} \backslash v$ is also a connected dominating set, which contradicts with the assumption that $D^{o p t}$ is an optimal solution. Thus, $v \notin D^{o p t}$.

For a given graph $G=(V, E)$, the set of vertices $V$ is divided into three sets $V_{\text {in }}, V_{\text {out }}$ and $V_{\text {cand }}$.

- $V_{\text {in }}$ consists of all articulation vertices of $G$ calculated by Tarjan's algorithm (Hopcroft \& Tarjan, 1973) , i.e., $V_{i n}=A(G)$. These vertices exist in each feasible solution;

- $V_{\text {out }}$ denotes a vertex set containing vertices from any clique $C \subseteq V$, which only connect to vertices in $C$ according to the Proposition 1. These vertices are not contained in any optimal solution;

- $V_{\text {cand }}=V \backslash\left(V_{\text {in }} \cup V_{\text {out }}\right)$ is the remaining vertex set.

Vertices in $V_{\text {in }}$ and $V_{\text {out }}$ are fixed in and excluded from optimal solutions, respectively. By excluding the vertices in $V_{\text {out }}$ and forcing to select vertices in $V_{\text {in }}$ for construction, the solution will be constructed from a reduced subset of $V$. Here, we propose a corresponding reasoning rule as follows.

Reasoning Rule for Construction: When picking vertex to construct an initial solution, the vertices in $V_{\text {out }}$ should not be chosen to be added and the vertices in $V_{\text {in }}$ should be selected with higher priority than those in $V_{\text {cand }}$.

\subsection{The 1hopReason Method}

Based on the reasoning rule above, we propose a novel construction algorithm named 1hopReason, to generate a high-quality initial solution. The psuedo-code of 1hopReason is shown in Algorithm 1. The 1hopReason algorithm contains a preprocessing method to obtain three vertex sets (lines 1-6) and a local greedy construction method using the reasoning rule for construction (lines 7-14).

At the beginning, Tarjan's algorithm (Hopcroft \& Tarjan, 1973) is used to obtain an articulation vertex set $A(G)$ as $V_{\text {in }}$ (line 1 ), while $V_{\text {out }}, V_{\text {cand }}$ and $D$ are initialized to $\emptyset$ (line 2 ). In lines 3-6, $V_{\text {out }}$ and $V_{\text {cand }}$ are generated iteratively by examining the vertices in $V \backslash V_{\text {in }}$. 


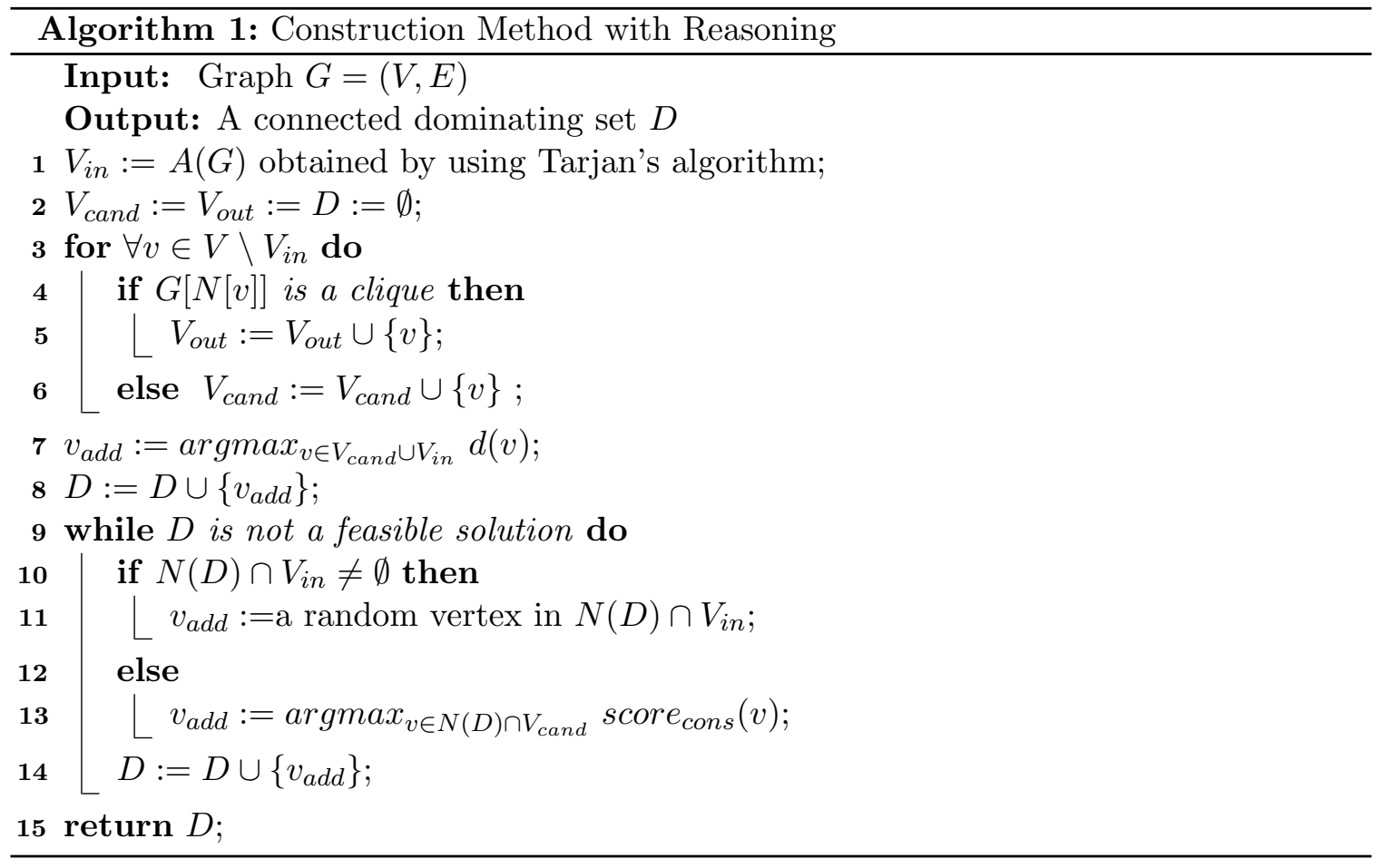

During each iteration, the algorithm checks whether $N[v]$ is a clique ${ }^{1}$ (line 4 ), and if this is the case, then the vertex is added to $V_{\text {out }}$ (line 5 ). If $N[v]$ is not a clique, $v$ is put into $V_{\text {cand }}$ (line 6). To accelerate the generating process, if the algorithm finds a vertex $v$ satisfying the condition, i.e., $G[N[v]]$ is a clique, all vertices with the same degree as $v$ in this clique are put into $V_{\text {out }}$ as they meet the condition as well, and the other vertices in $N[v]$ are directly put into $V_{\text {cand }}$. Besides, vertices that have been already assigned to $V_{\text {out }}$ or $V_{\text {cand }}$ will no longer be visited. The complexity of lines 3-6 is $O\left(\sum_{v \in V} \sum_{i \in N[v]} d(i)\right)=O\left(|V| \times \Delta_{G}^{2}\right)$.

The construction process starts from a set $D$ containing a vertex with the maximum degree (lines 7-8). At each step, it takes all 1-hop information into account using the reasoning rule. Specifically, if there exists a vertex $v_{a d d}$ in $N(D) \cap V_{i n}$, the algorithm will choose it first (lines 10-11). Otherwise, the algorithm picks a vertex $v$ in $N(D) \cap V_{\text {cand }}$ with the largest $\operatorname{score}_{\text {cons }}(v)$, which means the number of newly dominated vertices by adding $v$, breaking ties randomly (Cai, Hou, Wang, Luo, \& Lin, 2020) (line 13). The complexity of lines 7-14 is $O\left(|V| \times \Delta_{G}\right)$. Figure 1 gives an example for the construction method.

\section{Hybrid Dynamic Connectivity Maintenance Method}

In order to handle the performance bottleneck caused by maintaining connectivity in MCDS problem, we introduce a hybrid dynamic connectivity maintenance method (HDC+ for short). After reviewing two main previous connectivity maintenance methods, we propose our novel tree-based connectivity maintenance method, and finally introduce the HDC+

1. It is implemented by checking whether the degree in the induced subgraph $G[N[v]]$ of each vertex in $N[v]$ are all equal to $|N(v)|$. The complexity for checking a vertex $v$ is $\mathrm{O}\left(\sum_{i \in N[v]} d(i)\right)$. 


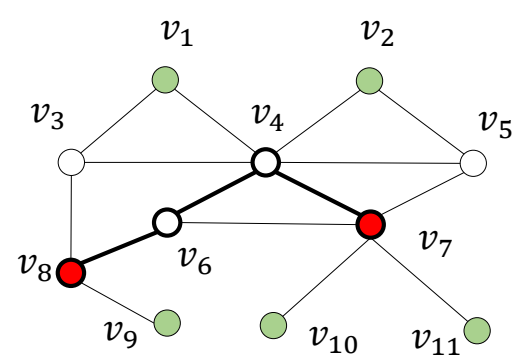

Figure 1: An example of the construction method. Firstly, the vertices set $V$ is divided into three sets, namely $V_{\text {in }}, V_{\text {out }}$ and $V_{\text {cand }}$, coloring red, green and white respectively. All articulation vertices are selected into $V_{i n}$. There are 5 cliques(vertex set $\left\{v_{1}, v_{3}, v_{4}\right\}$, $\left\{v_{2}, v_{4}, v_{5}\right\},\left\{v_{8}, v_{9}\right\},\left\{v_{7}, v_{10}\right\}$ and $\left.\left\{v_{7}, v_{11}\right\}\right)$ where at least one vertex could be selected into $V_{\text {out }}$ according to Proposition 1 . Then, the algorithm will choose $v_{4}$, with the maximum degree, as the first vertex of $D$. After that, $v_{7}$ is selected because it is the only vertex in $N(D) \cap V_{i n}$. For the next step, $v_{6}$ is chosen randomly from $\left\{v_{3}, v_{6}\right\}$ because they maximize the number of newly dominated vertices. After selecting $v_{8}, D$ becomes a feasible solution.

method. For convenience of discussions on complexity, we will use notations $m=\left|V_{D}\right|$ and $n=\left|E_{D}\right|$, where $G[D]=\left(V_{D}, E_{D}\right)$ is the induced subgraph of current candidate solution $D$.

\subsection{Previous Connectivity Maintenance Methods}

Before introducing $\mathrm{HDC}+$, we review two previous methods to handle the connectivity constraint, namely the substraction-based and addition-based methods.

The substraction-based method (SUB for short) is used by previous state-of-the-art MCDS algorithms such as RNS-TS (Wu et al., 2017). Moreover, SUB is applied to solve the weighted version of MCDS problem (Dagdeviren, Aydin, \& Cinsdikici, 2017). In order to keep the connectivity of candidate solution $D$, during each iteration of local search, the algorithm maintains the candidate removal vertex set, defined as $\operatorname{candRemoval}(D)=D \backslash$ $A(G[D])$. The traditional approach to computing $A(G[D])$ is called Tarjan's algorithm (Hopcroft \& Tarjan, 1973). It works as follows: depth first search (DFS for short) is used to determine whether the child vertex of a vertex $u$ can access the ancestor vertex of $u$ without passing through $u .^{2}$ If so, then $u$ is not an articulation vertex, and otherwise it is an articulation vertex. The complexity of the substraction-based method is $O(m+n)$.

The addition-based method has two versions. The first version, used in ACO-RVNS (Bouamama et al., 2019) and in MCDS/TS (Morgan \& Grout, 2007), works as follows: during each iteration, the algorithm starts from an empty candidate solution $D$, and iteratively adds a vertex from $N(D)$ to $D$ until $D$ becomes a feasible solution. The second version, used in GRASP (Li et al., 2017) and MSLS (Li et al., 2019), works as follows: the algorithm allows removing articulation vertices of $D$, so $D$ may become disconnected. Thus, before vertex $u$ is selected to be added, DFS is used to calculate the number of con-

2. The child vertex of $u$ is the vertex visited directly after $u$, while father and ancestor vertices of $u$ are the vertices visited directly and indirectly before $u$ by DFS. 
nected subgraphs of $D$ that $v \in N(u)$ belongs to, and the vertex with the largest number is preferred. The complexity of both versions is $O(m)$.

The complexity of the above two methods is at least $O(m)$. This makes algorithm time-consuming when applied to massive graphs, hindering the performance on massive graphs.

\subsection{Tree-Based Connectivity Maintenance Method}

To lower the time complexity of connectivity maintenance, we present a novel tree-based connectivity maintenance (TBC for short) method, which is inspired by spanning trees.

For this purpose, we first introduce the definitions of a spanning tree and a leaf vertex. Given a connected graph $G=(V, E)$, a spanning tree $T=\left(V^{\prime}, E^{\prime}\right)$ is defined as a connected subgraph of $G$ with $V^{\prime}=V, E^{\prime} \subseteq E$, without any cycles. Given a spanning tree $T=$ $\left(V^{\prime}, E^{\prime}\right)$, a vertex $v \in V^{\prime}$ is called a leaf vertex if $d_{T}(v)=1$, where $d_{T}(v)$ denotes the degree of $v$ in $T$, and the leaf set is defined as $L S(T)=\left\{v \mid d_{T}(v)=1, v \in V^{\prime}\right\}$.

Proposition 2 Given a spanning tree $T=\left(V^{\prime}, E^{\prime}\right)$ and its corresponding leaf set $L S(T)$ of graph $G=(V, E), L S(T)$ is a subset of $V \backslash A(G)$.

Proof: For any vertex $v \in L S(T)$, after removing $v$ and relevant edges through $v$ from $T$, the remaining graph is still a spanning tree of $G[V \backslash\{v\}]$. So $G[V \backslash\{v\}]$ remains connected, which means that $v$ is not an articulation vertex of $G$, inducing that $v \in V \backslash A(G)$. Thus, we can conclude that $L S(T) \subseteq V \backslash A(G)$.

Based on the definition and proposition above, we describe TBC as follows. Given a candidate solution $D$, a spanning tree $T$ of $G[D]$ and its corresponding leaf set $L S(T)$ are maintained during the search process. Each vertex $v \in L S(T)$ is allowed to be removed from $D$. All other vertices are forbidden to be removed. In contrast to the previous substraction-based method, given a current solution $D$, TBC calculates an approximate candRemoval $(D)=L S(T)$, which is a subset of $D \backslash A(G[D])$. In order to dynamically update candRemoval $(D)$, three updating rules of TBC are described as follows.

Construction Rule: Both $T$ and $L S(T)$ with respect to $G[D]$ are constructed according to the candidate solution $D$ by using breadth first search. The construction method is to set a randomly picked vertex in $D$ as the root of $T$ at first, and to iteratively expand $T$ by a randomly picked vertex in current $L S(T)$ until all vertices have been visited. When a vertex $v$ is picked, for each vertex $u \in(N(v) \cap D) \backslash T$, $v$ is set to the father vertex of $u$, and $u$ is added to $T$ and the $L S(T)$ should be updated accordingly.

Removing Rule: When vertex $v$ is selected to be removed, its father vertex $u$ needs to be found. If $d_{T}(u)=2$, meaning that $u$ becomes a leaf vertex after removing $v, L S(T)=$ $L S(T) \backslash\{v\} \cup\{u\}$. Otherwise, $L S(T)=L S(T) \backslash\{v\}$.

Adding Rule: When vertex $v$ is added to the candidate solution $D$, one vertex $u \in N(v) \cap T$ needs to be selected as the father vertex of $v$. In order to render larger candRemoval $(D)$, we prefer not to select a leaf vertex in $L S(T)$, and thus we pick the vertex $u$ with the maximum $d_{T}(u)$ among $\forall u \in N(v) \cap T$. If $u$ is a leaf vertex, $L S(T)=$ $L S(T) \backslash\{u\} \cup\{v\}$, else $L S(T)=L S(T) \cup\{v\}$. Lastly, $v$ is set as the child of $u$ in $T$.

The complexity of the construction rule used to construct and reconstruct the spanning tree is $O(m+n)$. When adding a vertex $v$ based on the Adding Rule, $N(v)$ has to be 


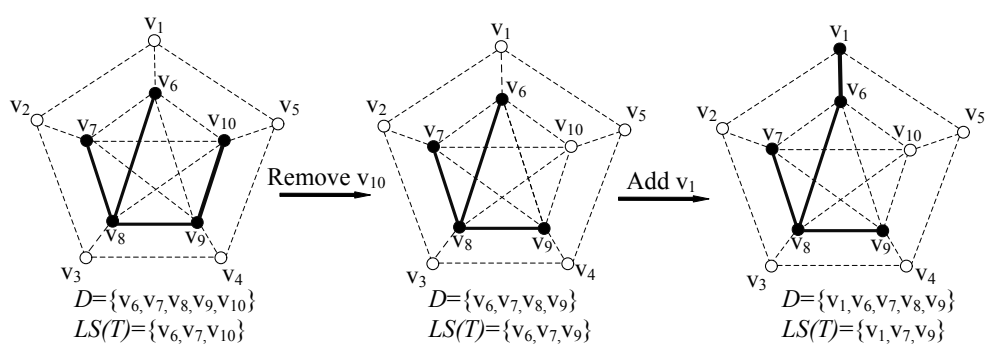

Figure 2: An example of the TBC method. (The solid nodes denote those vertices in the candidate solution $D$, while the solid edges compose the spanning tree $T$ of $G[D]$.)

searched to update $T$ and $L S(T)$, which has a complexity of $O\left(\Delta_{G}\right)$. When removing a vertex $v$ based on the Removing Rule, only the father vertex of $v$ needs to be searched to update $T$ and $L S(T)$, which has a complexity of $O(1)$. To make the TBC method more comprehensive, we present its example in Figure 2 regarding to removing $v_{10}$ and then adding $v_{1}$.

\subsection{The HDC Method and Its Improved Version HDC+}

For large graphs, TBC is substantially faster than previous connectivity maintenance methods. However, since TBC does not consider all candidate removal vertices, it may miss some high-quality options. As an extreme example, a candidate solution $D$ with a vertex $v \in D$ such that $D \backslash\{v\}$ is also a connected dominating set. However, TBC does not consider this option if $v$ is not a leaf vertex of the current spanning tree. In contrast, the substraction-based method using Tarjan's algorithm potentially considers more options for removal because it accurately determines the complete candidate removal vertex set, but its complexity is higher.

In order to take profit from the respective advantages of both methods, a balance must be achieved between the complexity and the accuracy of determining the candidate removal vertex set. We propose a heuristic called hybrid dynamic connectivity maintenance method (HDC) to switch between the two methods. Furthermore, we proposed an improved version called $\mathrm{HDC}+$, by reconstructing the spanning tree when falling into local minima for certain steps.

We first define four parameters as below: 1) parameter NoImpr denotes the maximum number of steps without improving the candidate solution, where NoImpr $\in[$ MinNoImpr, MaxNoImpr]; 2) parameter MinNoImpr denotes the minimum value of NoImpr, and it is also adopted as the absolute value of the change on NoImpr in each step; 3) parameter MaxNoImpr denotes the maximum number of steps using the current connectivity maintenance method; 4) parameter reconsGap denotes the number of non-improving steps in order to activate a tree reconstruction process.

Considering the difference of complexity between the two connectivity maintenance methods, two sets of parameters are respectively set for SUB and TBC.

Algorithm 2 gives a formal description of the HDC and HDC+ heuristics. stepNoImpr and stepOneCon denote the number of non-improving steps and the number of steps adopting one current connectivity maintenance method, respectively. 


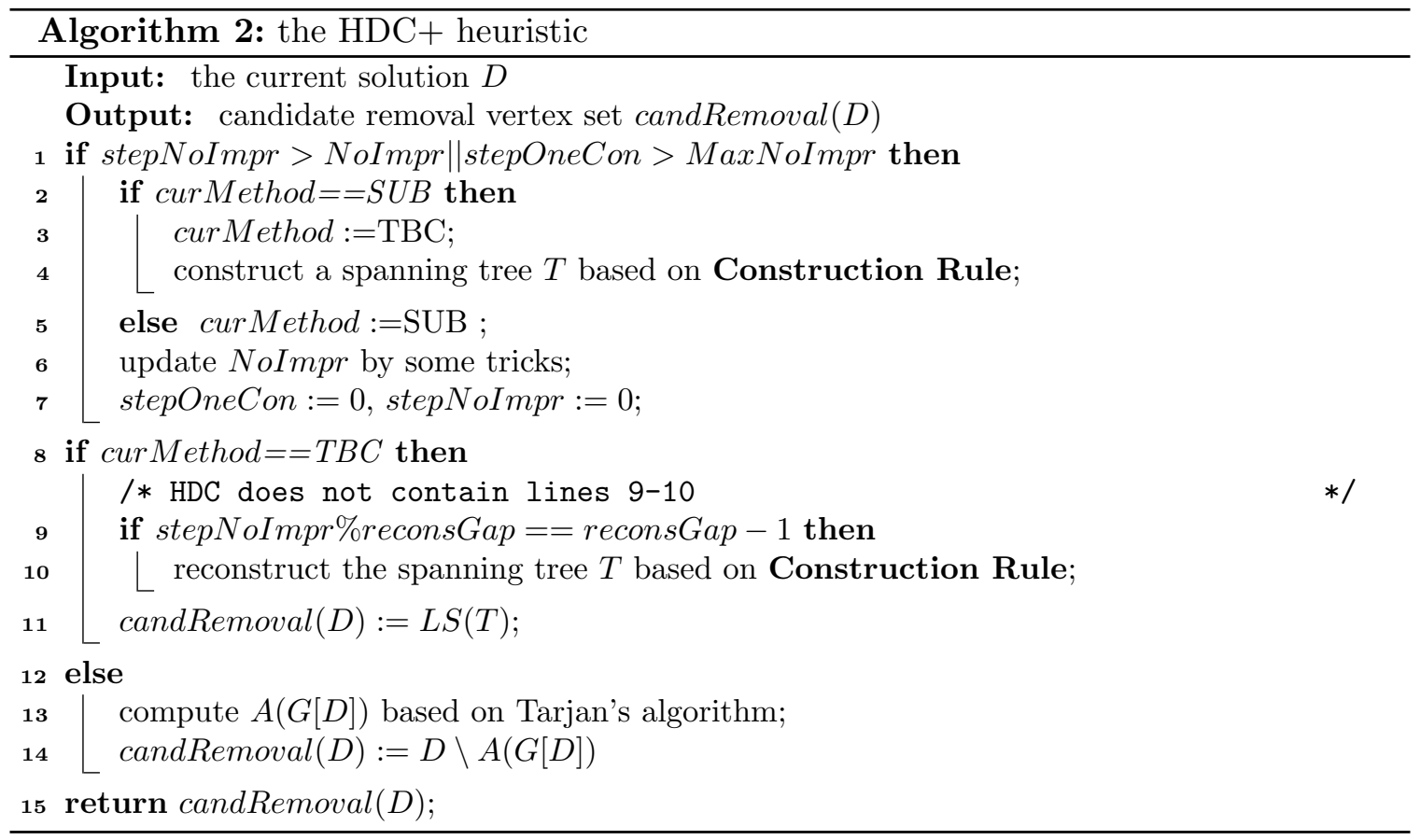

When the candidate solution has not been improved for NoImpr iterations or the current method has been used for MaxNoImpr iterations (line 1), the algorithm switches from the current connectivity maintenance method to the other one (lines 2-5). In particular, after switching to TBC, a spanning tree $T$ of $D$ and its corresponding leaf set $L S(T)$ need to be constructed (line 4). After the switching operation, NoImpr needs to be updated (line 6). Specifically, if stepNoImpr $>$ NoImpr, then the algorithm increases NoImpr by MinNoImpr so that it can search more exhaustively for better candidate solutions. Otherwise, if stepOneCon > MaxNoImpr, the algorithm decreases NoImpr by MinNoImpr in order to accelerate the search. Both stepNoImpr and stepOneCon should be reset to 0 (line 7). If the current method is TBC and the reconstruction condition is satisfied (line 9 ), then the spanning tree $T$ will be reconstructed (line 10), which is the main difference between HDC and HDC+ heuristics. Lastly, the algorithm uses the selected connectivity maintenance method to calculate the candidate removal vertex set candRemoval $(D)$ and returns it (lines 8-15).

\section{Two-Level Vertex Selection Heuristic}

Local search algorithms typically use scoring functions to choose an operation to execute in each step. In this section, we introduce a two-level vertex selection heuristic for local search MCDS algorithms. While the primary scoring function for vertex selection is commonly used for MCDS problem, we propose a secondary scoring function to break ties when the vertices have the same value of the primary function. Note that we use an incremental evaluation method, which is the same as FastDS (Cai et al., 2020), for our scoring function. 


\subsection{The Primary Scoring Function}

In the context of the MCDS problem, a scoring function is used to choose a vertex $u \in D$ for removal and a vertex $v \in N(D)$ for addition. Our algorithm adopts the frequency based scoring function (Wang et al., 2017) as the primary function for this purpose.

For each vertex $v \in V$, the frequency of $v$ is denoted as freq[v], which measures the number of steps that the vertex is not dominated. It can be simply calculated: in the beginning, freq[v] $=1$ for $\forall v \in V$; then, at the end of each iteration of local search, freq $[v]=$ freq $[v]+1$ for each non-dominated vertex $v \in V$. The frequency based scoring function, denoted as score, is defined as follows:

$$
\operatorname{score}(v)= \begin{cases}\sum_{u \in C_{1}} \text { freq }[u], & v \notin D \\ -\sum_{u \in C_{2}} \text { freq }[u], & v \in D\end{cases}
$$

where $C_{1}$ is the set of non-dominated vertices that would become dominated by adding $v$ to $D$ (the current candidate solution), and $C_{2}$ is the set of dominated vertices that would become non-dominated by removing $v$ from $D$.

\subsection{The Chrono-Safety Function}

In addition to considering the score value, we also observe that some dominated vertices are more "endangered" than others, which means that they may become non-dominated more easily. An extreme case is where a vertex is only dominated by one vertex (either by itself or by one of its neighbors). Such vertices may become non-dominated due to one exchanging step. Based on this consideration, we define the safety of vertices, taking into account the differences among dominated vertices. We first give the definition of the domination degree.

Definition 2 Given a connected graph $G=(V, E)$ and a candidate solution $D$, the domination degree of vertex $v$ is defined as $d d(v)=|N[v] \cap D|$, for each vertex $v \in V$.

This means that a vertex $v$ with $d d(v)=k$ is dominated by $k$ vertices. Thus, the larger the domination degree, the safer the corresponding vertex. We define a property of vertices named safety. The safety of a vertex $v$, denoted as $s f(v)$, is $d d(v)$ if $v \notin D$, or $-d d(v)$ if $v \in D$. Note that we introduce a negative sign for the case $v \in D$ just for the convenience that in both cases we always prefer larger safety value.

The safety-based ideas are inspired by the concept of subscore for the SAT problem (Cai $\& \mathrm{Su}, 2013)$ which considers the satisfaction degree of clauses. Moreover, as far as we know, it is the first time that the definition of safety is applied to solve a graph optimization problem.

Besides safety, we also consider the additional property of vertex (i.e., age) to break ties for the sake of diversification. The age of a vertex $v$ is defined as the number of steps since $v$ was last chosen. The default value of age for those vertices never chosen before are set to CurStep, where CurStep is the number of current steps during local search. During the search process, the algorithm prefers to select a vertex with larger age value for diversity.

Combining the two factors above, namely safety and age, our secondary scoring function is defined as the chrono-safety of a vertex $v$, denoted as $c s(v)$.

$$
c s(v)= \begin{cases}\text { age }(v) \times s f(v), & v \notin D \\ (\text { CurStep }- \text { age }(v)) \times s f(v), & v \in D\end{cases}
$$


In the above $c s$ function, age dominates the selection strategy if there exist candidate vertices which have not been chosen for a relatively long time. Meanwhile, safety dominates the selection strategy if vertices in candidate selection set are all recently chosen. Note that by introducing CurStep for the case $v \in D$, we always prefer larger $c s$ for both adding and removal.

\subsection{Vertex Selection Rule in Our Algorithm}

As introduced in Section 2, the BMS heuristic is a common technique in picking a goodquality element from a large candidate set, which is usually used in local search for large combinatorial optimization instances. In this work, we employ a variant of BMS, which gives higher priority to the important vertices (those in $V_{\text {in }}$ and $V_{\text {out }}$ ) by giving them higher probability when sampling. By using the score function as the primary scoring function and the chrono-safety function as the secondary function, we design a two-level vertex selection heuristic based on BMS for our local search algorithm. It can be described as below.

Vertex Selection Rule: Sample the given candidate set for $k$ times. A vertex in $V_{\text {in }}$ (resp. $V_{\text {out }}$ ) is sampled twice when picking vertex to add (resp. remove). Return the sampled vertex $v$ with the greatest $\operatorname{score}(v)$, breaking ties by preferring the one with the greatest $c s(v)$. In our work, the BMS parameter $k$ is set to 100 .

In our algorithm, we employ the novel vertex selection rule to decide which vertices to be added or removed. To avoid visiting previous candidate solutions, we use the $\mathrm{CC}^{2}$ strategy (Wang et al., 2017) in the adding process, and use the tabu strategy (Glover \& Laguna, 1998) recording the adding operations to prevent removing a just added vertex for the next $t t$ iterations. In our work, $t t=3$. There exist an extreme case that all sampled vertices are forbidden by tabu or $\mathrm{CC}^{2}$ strategies. For this case, the algorithm picks a random vertex from the sampled vertices. In contrast to previous BMS versions which mainly focus on adjusting the $k$ value, the novelty mind of our BMS strategy is to optimize the sample procedure by giving a higher probability for special vertices. Specifically, the selection probability of vertices in $V_{\text {in }}$ and $V_{\text {out }}$ is twice that of vertices in $V_{\text {cand }}$.

\section{A New Local Search Algorithm for MCDS Problem}

Based on HDC+ and the chrono-safety vertex selection heuristic, we develop a local search algorithm for the MCDS problem called FastCDS. The pseudo-code of FastCDS is shown in Algorithm 3.

In the beginning, FastCDS initializes score, sf and age (line 1), and sets cur Method = TBC which means that the TBC method is selected as the initial connectivity maintenance method (line 2). The non-improvement step stepNoImpr and the number of steps used by the current connectivity maintenance method stepOneCon are both set to 0 (line 2). Then, the algorithm constructs the initial candidate solution $D$ using the proposed 1hopReason method (line 3). According to the construction rule, the algorithm builds a spanning tree $T$ of $D$ (line 4$)$, and then the candidate removal vertex set candRemoval $(D)$ is initialized as $L S(T)$ (line 5).

During the local search procedure (lines 6-19), the algorithm first uses the HDC+ heuristic to update candRemoval $(D)$ (line 7 ). If $D$ is a feasible solution, which means that 


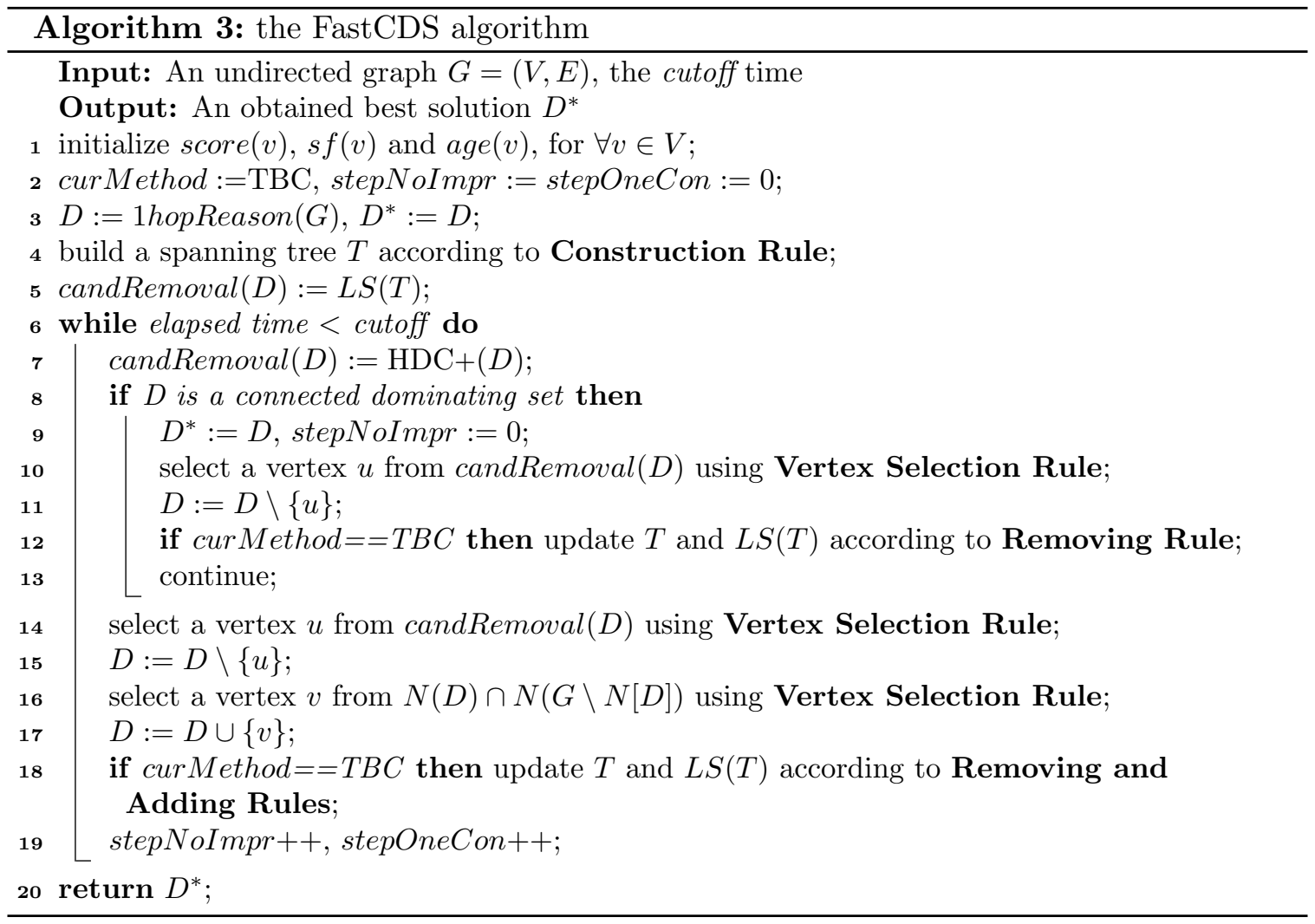

the algorithm has already found a connected dominating set of size $|D|, D^{*}$ is updated to $D$ and stepNoImpr is set to 0 (line 9). Then, the algorithm continues to find a solution of size $(|D|-1)$, i.e., by removing a vertex $v$ from $D$ using the vertex selection rule (lines 10-11). If the algorithm selects the TBC method as the current connectivity maintenance method, the corresponding spanning tree $T$ and its leaf set $L S(T)$ should be updated based on the removing rule (line 12).

If $D$ is an infeasible solution, the algorithm tries to exchange two vertices (lines 14-17), i.e., removing a non-tabu vertex $u$ chosen from candRemoval $(D)$ via the vertex selection rule, and then adding a vertex $v \in N(D) \cap N(G \backslash N[D])$ to $D$ via the $C C^{2}$ strategy and the selection rule, where $G \backslash N[D]$ is the non-dominated vertex set and $N(D)$ contains vertices maintaining connectivity. Finally, the algorithm needs to update $T, L S(T)$, stepOneCon, and stepNoImpr accordingly (lines 18-19). When the time limit is reached, the best solution found $\left(D^{*}\right)$ will be returned. The complexity of each iteration in the local search process is $\mathrm{O}(|D|)$ or $\mathrm{O}\left(\Delta_{G}\right)$ when using SUB or TBC methods respectively.

Moreover, we make use of a trick for the HDC+ heuristic. If $|D|<100$ (that is, if the complexity of the SUB method is acceptable), using TBC becomes trivial and thus we only use the SUB method under such circumstance. 


\subsection{The Differences Between FastCDS and NuCDS}

An early version of FastCDS called NuCDS has been published in the conference version (Li et al., 2020a). There are three main differences between FastCDS and NuCDS.

- FastCDS adopts an effective construction algorithm based on the reasoning rule namely 1 hopReason to generate an initial solution, while NuCDS uses the previous greedy construction algorithm mentioned in (Khuller \& Yang, 2019).

- FastCDS improves HDC method proposed in NuCDS, resulting in the HDC+ method. Specifically, when falling into the local minima for certain steps, HDC+ will reconstruct the spanning tree to diversify the search space.

- FastCDS and NuCDS use different selection rules. They combine the age property with safety in different manners. Also, FastCDS assigns different selection priorities to different candidate vertices in $V_{\text {in }}, V_{\text {out }}$ and $V_{\text {cand }}$.

\section{Experimental Evaluation}

In this section, we carry out extensive experiments to evaluate the effectiveness of FastCDS. We first introduce the benchmarks, the experiment setup and reporting methodology, so that the readers can understand the experimental parts more easily.

\subsection{Benchmarks and Experiment Methodologies}

For our experiments, we adopt several popular benchmarks, mainly divided into two groups, including classic benchmarks and massive graphs. We divide classic benchmarks into five groups: the instances of the maximum leaf spanning tree problem (Lucena et al., 2010), bus power flow test cases $^{3}$, random geometric graphs (Jovanovic \& Tuba, 2013), and random graphs from (Erdem et al., 2009) and from (Bouamama et al., 2019). In total, 121 classic instances are considered to evaluate the performance of FastCDS.

We also evaluated the algorithms on massive graphs, including massive real-world graphs from the Network Data Repository (NDR) (Rossi \& Ahmed, 2015) and Stanford Large Network Dataset Collection (SNAP) ${ }^{4}$, as well as large instances from the 10th DIMACS implementation challenge (DIMACS10) ${ }^{5}$. As in previous work (Lin et al., 2017), we only report the results on graphs from the SNAP and DIMACS10 benchmarks with at least 30,000 vertices (with the exception of a few cases with fewer vertices). Directed edges are considered as undirected edges if the instance is directed, and the maximal connected component is chosen if the instance is disconnected, so all instances are undirected connected. We do not report the results on graphs from the NDR benchmark with fewer than 100,000 vertices or fewer than 1,000,000 edges. Hence, we picked 22, 31 and 65 instances from SNAP, DIMACS10, and NDR benchmarks respectively, leading to totally 118 massive graphs.

3. http://labs.ece.uw.edu/pstca

4. http://snap.stanford.edu/data

5. https://www.cc.gatech.edu/dimacs10/ 


\begin{tabular}{lll}
\hline Parameter & Domain & Chosen value \\
\hline MinNoImpr for SUB & $\{100,1000\}$ & 100 \\
MinNoImpr for TBC & $\{10000,100000,1000000\}$ & 100000 \\
MaxNoImpr for SUB & $\{2,5,10\} \times$ MinNoImpr for SUB & $2 \times$ MinNoImpr for SUB \\
MaxNoImpr for TBC & $\{2,5,10\} \times$ MinNoImpr for TBC & $10 \times$ MinNoImpr for TBC \\
NoImpr for SUB & $\{1,2,5\} \times$ MinNoImpr for SUB & $1 \times$ MinNoImpr for SUB \\
NoImpr for TBC & $\{1,2,5\} \times$ MinNoImpr for TBC & $2 \times$ MinNoImpr for TBC \\
reconsGap & $\{500,1000\}$ & 500 \\
\hline
\end{tabular}

Table 1: Parameter tuning for the preliminary experiments.

We compare FastCDS with five state-of-the-art heuristic algorithms: MSLS (Li et al., 2019), ACO-RVNS (Bouamama et al., 2019), ACO-efficient ${ }^{6}$, RNS-TS (Wu et al., 2017) and $\mathrm{NuCDS}$ (Li et al., 2020a). The codes of all competitors were kindly provided by the authors. RNS-TS was implemented in Java while FastCDS and the other competitors were implemented in $\mathrm{C}++$ and complied by $\mathrm{g}++$ with '-O3'. Data structures of all competitors were modified to handle massive graphs. All experiments were run on a server with Intel Xeon Platinum 8153 @2.00GHz with 512GB RAM under Centos 7.7.1908.

All algorithms were executed 10 times on each instance independently. The cutoff time was set to 1000 seconds for the classic benchmarks, and 3600 seconds for massive graphs.

The following information is reported: the best size ( $\mathrm{min})$, the average size $(\mathrm{avg})$ of the solutions found over the 10 runs. The best min and avg found among the algorithms are shown in bold. If an algorithm fails to find a solution within the time limit, the results are marked as 'N/A'. We report the number of instances that get the best solution and the best average solution by \#min and \#avg. We also list the \#feasible information, denoted as the number of instances that a solver can find a feasible solution.

In preliminary experiments, we tried different parameters, and we found that HDC + is not very sensitive to parameter settings. The tried parameter values and chosen values are presented in Table 1.

\subsection{Results on Classic Benchmarks}

Most instances of classic benchmarks are so easy that all algorithms obtain the same solution quality very quickly. We ignore these easy instances, but report the average run time when all algorithms obtain the same minimal and average values in Figure 3, which shows the effectiveness of FastCDS. From this figure, FastCDS is more effective than its competitors, while performing a little worse than NuCDS on a few easy instances which can be solved less than 0.1 second. The results of the remaining 55 classic instances are shown in Table 2 . FastCDS obtains better solutions than NuCDS, MSLS, ACO-efficient, ACO-RVNS and RNS-TS on 16, 32, 36, 22 and 29 instances respectively, while FastCDS fails to match the solutions obtained by some competitors only on 5 instances. Among the instances where FastCDS generates a solution with the same value as the best competitor, FastCDS obtains better average size on 35 instances with only 3 exceptions.

6. The authors would like to thank Christian Blum, the author of ACO-RVNS, for providing the improved version called ACO-efficient, which is specialized for massive graphs and performs better than ACORVNS on most large instances. 


\begin{tabular}{|c|c|c|c|c|c|c|c|c|c|c|c|c|}
\hline \multirow{2}{*}{ Instance } & \multicolumn{2}{|c|}{ FastCDS } & \multicolumn{2}{|c|}{$\mathrm{NuCDS}$} & \multicolumn{2}{|c|}{ MSLS } & \multicolumn{2}{|c|}{$\mathrm{ACO}_{e}$} & \multicolumn{2}{|c|}{$\mathrm{ACO}$} & \multicolumn{2}{|l|}{ RNS } \\
\hline & $\min$ & avg & $\min$ & avg & $\min$ & avg & $\min$ & avg & $\min$ & avg & $\min$ & avg \\
\hline v150_d10 & 14 & 14 & 14 & 14 & 14 & 14.6 & 14 & 14.6 & 14 & 14.5 & 14 & 14 \\
\hline ieee_300_bus & 129 & 129 & 130 & 130.9 & 129 & 129.2 & 129 & 129.2 & 129 & 129 & 129 & 129 \\
\hline n1000_200_r100 & 38 & 38 & 38 & 38 & 39 & 39.6 & 39 & 39.1 & 38 & 38.2 & 38 & 38.5 \\
\hline n1000_200_r110 & 34 & 34 & 34 & 34 & 34 & 34.1 & 34 & 34.1 & 34 & 34 & 34 & 34 \\
\hline n1000_200_r120 & 29 & 29 & 29 & 29 & 29 & 30.3 & 30 & 30.2 & 29 & 29 & 29 & 29 \\
\hline n1000_200_r130 & 26 & 26 & 26 & 26 & 26 & 26.8 & 26 & 26.7 & 26 & 26 & 26 & 26 \\
\hline n1000_200_r140 & 23 & 23 & 23 & 23 & 23 & 23.1 & 23 & 23.4 & 23 & 23 & 23 & 23 \\
\hline n1000_200_r150 & 21 & 21 & 21 & 21 & 21 & 21.1 & 21 & 21.1 & 21 & 21 & 21 & 21 \\
\hline n1000_200_r160 & 19 & 19 & 19 & 19 & 19 & 19.6 & 36 & 36.3 & 19 & 19 & 19 & 19.1 \\
\hline n1500_250_r130 & 49 & 49 & 49 & 49 & 49 & 50 & 49 & 49.7 & 49 & 49.1 & 49 & 49 \\
\hline n1500_250_r140 & 43 & 43 & 43 & 43.7 & 44 & 44.3 & 44 & 44 & 43 & 43.9 & 43 & 43.9 \\
\hline n1500_250_r150 & 40 & 40 & 40 & 40 & 41 & 41.6 & 41 & 41.6 & 40 & 40.7 & 40 & 40.1 \\
\hline n1500_250_r160 & 36 & 36 & 36 & 36 & 37 & 37.7 & 36 & 36.3 & 36 & 36 & 36 & 36 \\
\hline n2000_300_r200 & 41 & 41 & 41 & 41.5 & 43 & 43.1 & 42 & 42.5 & 42 & 42.1 & 41 & 41.6 \\
\hline n2000_300_r210 & 38 & 38 & 38 & 38 & 38 & 38.6 & 38 & 38.5 & 38 & 38 & 38 & 38 \\
\hline n2000_300_r220 & 35 & 35 & 35 & 35 & 36 & 36.3 & 35 & 36 & 35 & 35.1 & 35 & 35 \\
\hline n2000_300_r230 & 33 & 33 & 33 & 33 & 34 & 34.8 & 34 & 34.5 & 33 & 33 & 33 & 33.2 \\
\hline n2500_350_r200 & 59 & 59.4 & 60 & 60 & 61 & 61.3 & 61 & 62.3 & 60 & 60.7 & 60 & 60.3 \\
\hline n2500_350_r210 & 54 & 54 & 54 & 54.9 & 56 & 57 & 57 & 58.2 & 55 & 56.1 & 55 & 56 \\
\hline n2500_350_r220 & 51 & 51 & 51 & 51.1 & 52 & 54.3 & 54 & 54.7 & 51 & 52.7 & 51 & 51.4 \\
\hline n2500_350_r230 & 48 & 48 & 49 & 49.1 & 50 & 50.6 & 50 & 51.7 & 48 & 49.5 & 49 & 49 \\
\hline n3000_400_r210 & 74 & 74 & 74 & 74 & 74 & 76.3 & 76 & 76.6 & 74 & 75.5 & 75 & 75.1 \\
\hline n3000_400_r220 & 69 & 69.2 & 70 & 70 & 71 & 71.9 & 71 & 71.6 & 70 & 70.8 & 70 & 70.3 \\
\hline n3000_400_r230 & 64 & 64 & 64 & 64.8 & 66 & 67 & 65 & 67.1 & 65 & 65.9 & 65 & 65.1 \\
\hline n3000_400_r240 & 60 & 60 & 60 & 60.9 & 61 & 62.5 & 62 & 62.8 & 61 & 61.7 & 61 & 61.2 \\
\hline n600_100_r110 & 14 & 14 & 14 & 14 & 14 & 14.5 & 14 & 14.6 & 14 & 14 & 14 & 14 \\
\hline n700_200_r100 & 22 & 22 & 22 & 22 & 22 & 22.5 & 22 & 22.9 & 22 & 22 & 22 & 22 \\
\hline n700_200_r110 & 20 & 20 & 20 & 20 & 20 & 20 & 20 & 20.5 & 20 & 20 & 20 & 20 \\
\hline n700_200_r120 & 17 & 17 & 17 & 17 & 17 & 17 & 17 & 17.8 & 17 & 17 & 17 & 17 \\
\hline n700_200_r70 & 38 & 38 & 38 & 38.1 & 39 & 39.6 & 39 & 39 & 38 & 38.1 & 38 & 38.5 \\
\hline n700_200_r80 & 32 & 32 & 32 & 32 & 33 & 33 & 33 & 33 & 32 & 32 & 32 & 32 \\
\hline n1000_ep0007 & 179 & 179.4 & 179 & 179 & 191 & 194 & 187 & 189 & 185 & 186.6 & 189 & 190.7 \\
\hline n1000_ep0014 & 98 & 98.9 & 98 & 98 & 105 & 105.5 & 104 & 105.3 & 101 & 103.1 & 103 & 105.3 \\
\hline n1000_ep0028 & 59 & 59.6 & 59 & 59.9 & 62 & 62.5 & 62 & 62.8 & 61 & 62.8 & 63 & 63.6 \\
\hline n1000_ep0056 & 36 & 36.3 & 37 & 37 & 37 & 37 & 37 & 37.8 & 37 & 37.8 & 38 & 38.2 \\
\hline n1000_ep0112 & 21 & 21.6 & 22 & 22 & 22 & 22 & 22 & 22 & 22 & 22 & 22 & 22.1 \\
\hline n1000_ep0224 & 12 & 12 & 12 & 12.5 & 12 & 12 & 12 & 12.7 & 12 & 12 & 12 & 12.2 \\
\hline n1000_r0048 & 276 & 276.7 & 275 & 277.3 & 276 & 276.5 & 275 & 275.2 & 275 & 275.8 & 280 & 283.7 \\
\hline n1000_r0070 & 127 & 127.3 & 125 & 126.7 & 125 & 125 & 128 & 129.3 & 127 & 128.4 & 132 & 134.4 \\
\hline n1000_r0100 & 60 & 60.8 & 61 & 61.8 & 62 & 62.4 & 64 & 65.4 & 64 & 65.4 & 65 & 66.1 \\
\hline n1000_r0140 & 31 & 31.2 & 32 & 32.3 & 33 & 33 & 33 & 33.3 & 32 & 32.9 & 34 & 34.6 \\
\hline n1000_r0207 & 15 & 15 & 15 & 15.1 & 16 & 16 & 16 & 16.2 & 15 & 15.7 & 16 & 16.6 \\
\hline n1000_r0308 & 7 & 7 & 7 & 7 & 7 & 7 & 7 & 7 & 7 & 7 & 7 & 7.7 \\
\hline n5000_ep0007 & 275 & 276.3 & 264 & 265.6 & 277 & 277.4 & 277 & 278.2 & 277 & 278.2 & 392 & 424.8 \\
\hline n5000_ep0014 & 161 & 162.3 & 163 & 164.2 & 161 & 162.1 & 164 & 164.2 & 165 & 166.2 & 206 & 220 \\
\hline n5000_ep0028 & 95 & 95 & 94 & 94 & 95 & 95.1 & 96 & 96 & 95 & 95.8 & 121 & 25.8 \\
\hline n5000_ep0056 & 54 & 54.9 & 56 & 56 & 55 & 55 & 55 & 55.6 & 56 & 56.1 & 76 & 100 \\
\hline n5000_ep0112 & 31 & 31 & 32 & 32 & 31 & 31.4 & 31 & 31.9 & 31 & 31.7 & 2707 & 2934 \\
\hline n5000_ep0224 & 17 & 17 & 18 & 18 & 17 & 17 & 17 & 17 & 17 & 17.1 & 3888 & 4045 \\
\hline n5000_r0048 & 270 & 270.3 & 268 & 270 & 275 & 275.8 & 280 & 284.6 & 280 & 284.6 & 319 & 336.1 \\
\hline n5000_r0070 & 126 & 126 & 127 & 128.4 & 133 & 133.4 & 132 & 136.4 & 132 & 136.2 & 147 & 150.3 \\
\hline n5000_r0100 & 62 & 62 & 63 & 64.4 & 68 & 68.42 & 70 & 71.5 & 72 & 72.5 & 74 & 78.2 \\
\hline n5000_r0140 & 32 & 32.4 & 33 & 33.2 & 36 & 36.71 & 37 & 37.4 & 36 & 36.9 & 39 & 41 \\
\hline n5000_r0207 & 15 & 15.4 & 16 & 16 & 17 & 17 & 18 & 18.9 & 16 & 16.8 & 2099 & 2797.3 \\
\hline n5000_r0308 & 7 & 7 & 7 & 7.6 & 8 & 8.28 & 9 & 9.5 & 8 & 8 & 3652 & 3876 \\
\hline
\end{tabular}

Table 2: Results of FastCDS, NuCDS, MSLS, ACO-efficient, ACO-RVNS and RNS-TS on classic benchmarks. To save space, we denote ACO-efficient, ACO-RVNS and RNS-TS as $\mathrm{ACO}_{e}, \mathrm{ACO}$ and RNS.

\subsection{Results on Massive Benchmarks}

Table 3 compares FastCDS with its competitors on massive graphs, and shows the summarized results. The detailed results can be found in Tables 4 and 5. And we report the 


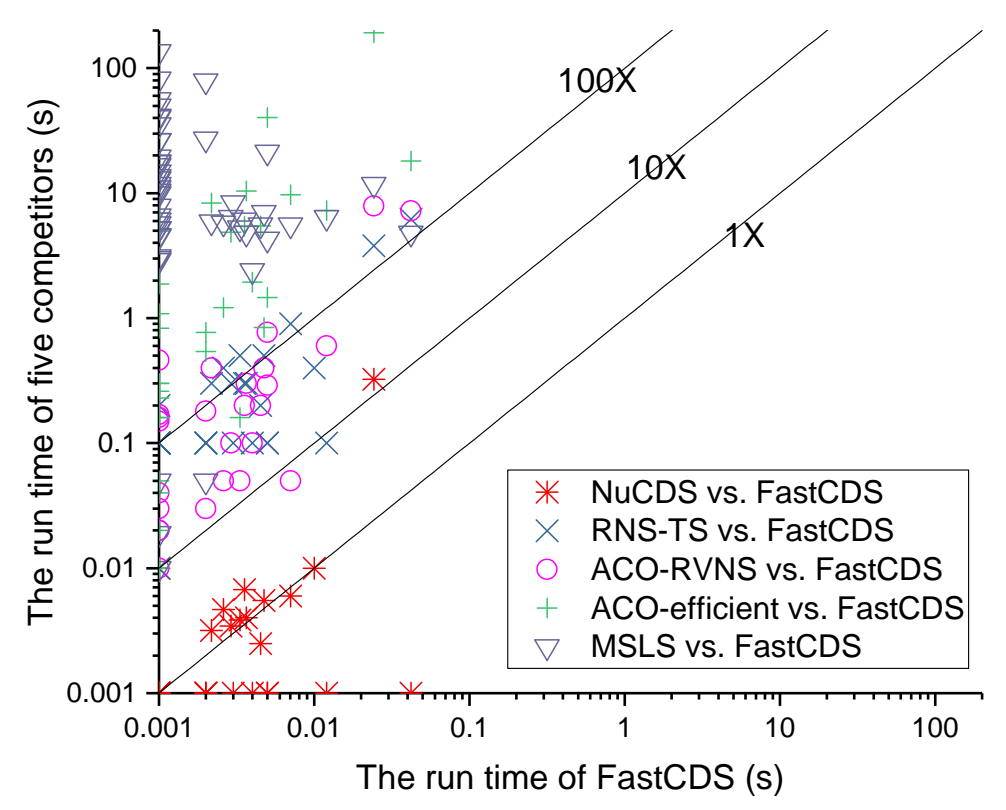

Figure 3: Average running time of FastCDS and competitors. The chosen instances are very easy that all algorithms obtain the same solution quality very quickly.

\begin{tabular}{llllllll}
\hline Benchmark & & FastCDS & NuCDS & MSLS & ACO $_{e}$ & ACO & RNS \\
\hline \multirow{3}{*}{ DIMACS10(22) } & \#feasible & 22 & 22 & 16 & 15 & 11 & 12 \\
& \#min & 19 & 4 & 4 & 3 & 1 & 0 \\
& \#avg & 20 & 4 & 2 & 1 & 0 & 0 \\
\hline \multirow{3}{*}{ SNAP(31) } & \#feasible & 31 & 31 & 14 & 15 & 5 & 4 \\
& \#min & 28 & 5 & 1 & 1 & 1 & 0 \\
& \#avg & 29 & 3 & 1 & 1 & 0 & 0 \\
\hline \multirow{3}{*}{ NDR(65) } & \#feasible & 65 & 61 & 15 & 18 & 2 & 10 \\
& \#min & 59 & 10 & 2 & 2 & 1 & 0 \\
& \#avg & 60 & 7 & 0 & 0 & 0 & 0 \\
\hline
\end{tabular}

Table 3: Summarized results of FastCDS, NuCDS, MSLS, ACO-efficient, ACO-RVNS and RNS-TS on DIMACS10, SNAP and NDR benchmarks.

time (in seconds) when finding the min cost in column time. FastCDS significantly outperforms all competitors. FastCDS obtains the best solution on 106 instances, and it is the only algorithm that can solve all these 118 instances within the time limit. In addition, we need to point out an error in our previous work (Li et al., 2020a) that we mistakenly took 4 instances which cannot be initialized within the time limit as solvable instances. Among all those solvable instances, the best solution values obtained by FastCDS are on average $2.26 \%, 2.64 \%, 3.0 \%, 2.79 \%$ and $79.08 \%$ smaller than those found by NuCDS, MSLS, ACO-efficient, ACO-RVNS, and RNS-TS, respectively. The excellent results of FastCDS on massive graph are mainly attributed to the effectiveness of our proposed strategies. 


\begin{tabular}{|c|c|c|c|c|c|c|c|c|c|c|c|}
\hline \multirow{2}{*}{ Instance } & \multicolumn{3}{|l|}{ FastCDS } & \multicolumn{3}{|l|}{ NuCDS } & \multicolumn{2}{|l|}{ MSLS } & \multirow{2}{*}{$\frac{\mathrm{ACO}_{e}}{\min }$} & \multirow{2}{*}{$\begin{array}{l}\mathrm{ACO} \\
\min \\
\end{array}$} & \multirow{2}{*}{$\begin{array}{l}\text { RNS } \\
\min \\
\end{array}$} \\
\hline & $\min$ & avg & time & $\min$ & avg & time & $\min$ & avg & & & \\
\hline Amazon0302 & 47458 & 47580.1 & 1.62 & 45815 & 45992.6 & 3190.61 & 48225 & 48625.6 & 47969 & $\mathrm{~N} / \mathrm{A}$ & N/A \\
\hline Amazon0312 & 51096 & 51153.9 & 1731.2 & 52077 & 52257.8 & 3522.06 & $\mathrm{~N} / \mathrm{A}$ & $\mathrm{N} / \mathrm{A}$ & $\mathrm{N} / \mathrm{A}$ & $\mathrm{N} / \mathrm{A}$ & $\mathrm{N} / \mathrm{A}$ \\
\hline Amazon0505 & 53029 & 53069.3 & 1632.52 & 54041 & 54125.7 & 3582.33 & $\mathrm{~N} / \mathrm{A}$ & $\mathrm{N} / \mathrm{A}$ & $\mathrm{N} / \mathrm{A}$ & $\mathrm{N} / \mathrm{A}$ & $\mathrm{N} / \mathrm{A}$ \\
\hline Amazon0601 & 46978 & 47012.6 & 1900.63 & 47963 & 48195.4 & 3275.92 & $\mathrm{~N} / \mathrm{A}$ & $\mathrm{N} / \mathrm{A}$ & $\mathrm{N} / \mathrm{A}$ & $\mathrm{N} / \mathrm{A}$ & $\mathrm{N} / \mathrm{A}$ \\
\hline Cit-HepPh & 3223 & 3225.1 & 3350.13 & 3263 & 3270 & 3508.45 & 3395 & 3407.2 & 3363 & 3417 & 31018 \\
\hline Cit-HepTh & 3177 & 3178.8 & 1177.47 & 3197 & 3204.7 & 2998.91 & 3289 & 3303.3 & 3264 & 3320 & 24036 \\
\hline cit-Patents & 686740 & 686969.8 & 86.58 & 734910 & 735877.2 & 2683.82 & $\mathrm{~N} / \mathrm{A}$ & $\mathrm{N} / \mathrm{A}$ & N/A & N/A & $\mathrm{N} / \mathrm{A}$ \\
\hline Email-EuAll & 2368 & 2368 & 1.07 & 2371 & 2371 & 5.45 & 2368 & 2368.6 & 2368 & 2373 & 224163 \\
\hline p2p-Gnutella04 & 2267 & 2267 & 0.99 & 2268 & 2270 & 2297.17 & 2294 & 2295.6 & 2270 & 2279 & 6526 \\
\hline p2p-Gnutella24 & 5468 & 5468 & 1.25 & 5471 & 5471 & 10.68 & 5475 & 5477.1 & 5469 & 5470 & 23819 \\
\hline p2p-Gnutella25 & 4556 & 4556 & 0.76 & 4558 & 4558 & 4.18 & 4561 & 4562.7 & 4556 & 4557 & 19467 \\
\hline p2p-Gnutella30 & 7226 & 7226 & 5.82 & 7229 & 7229 & 55.23 & 7238 & 7240.5 & 7229 & 7231 & 34578 \\
\hline p2p-Gnutella31 & 12672 & 12672.1 & 134.54 & 12674 & 12674.4 & 267.24 & 12677 & 12678.7 & 12677 & 12683 & 61061 \\
\hline Slashdot0811 & 14989 & 14989 & 1.54 & 14990 & 14990 & 37.81 & 14993 & 14994.5 & 14994 & $\mathrm{~N} / \mathrm{A}$ & 76148 \\
\hline Slashdot0902 & 16159 & 16159 & 35.61 & 16160 & 16160 & 138.27 & 16170 & 16173.81 & 16169 & $\mathrm{~N} / \mathrm{A}$ & 81006 \\
\hline soc-Epinions1 & 16667 & 16667 & 290.36 & 16667 & 16667 & 77.64 & 16668 & 16668.8 & 16671 & N/A & 74677 \\
\hline web-BerkStan & 30887 & 30898 & 2266.24 & 30967 & 30984.4 & 3542.31 & 31308 & 31332.3 & $\mathrm{~N} / \mathrm{A}$ & $\mathrm{N} / \mathrm{A}$ & N/A \\
\hline web-Google & 86882 & 86905.4 & 3079.25 & 86963 & 86992.3 & 3582.44 & $\mathrm{~N} / \mathrm{A}$ & $\mathrm{N} / \mathrm{A}$ & $\mathrm{N} / \mathrm{A}$ & $\mathrm{N} / \mathrm{A}$ & $\mathrm{N} / \mathrm{A}$ \\
\hline web-NotreDame & 25565 & 25586.2 & 2.21 & 25664 & 25665.8 & 824.37 & 25406 & 25462 & 25686 & 25641 & $\mathrm{~N} / \mathrm{A}$ \\
\hline web-Stanford & 11553 & 11557.2 & 1888.73 & 11581 & 11586.6 & 2351.61 & 11541 & 11600.3 & 11706 & 11742 & $\mathrm{~N} / \mathrm{A}$ \\
\hline WikiTalk & 35038 & 35038 & 8.86 & 35038 & 35038 & 132.11 & $\mathrm{~N} / \mathrm{A}$ & $\mathrm{N} / \mathrm{A}$ & $\mathrm{N} / \mathrm{A}$ & $\mathrm{N} / \mathrm{A}$ & $\mathrm{N} / \mathrm{A}$ \\
\hline Wiki-Vote & 1101 & 1101 & 0.09 & 1101 & 1101 & 0.48 & 1101 & 1101 & 1101 & 1101 & 3093 \\
\hline 333SP & 1186502 & 1186858.1 & 36.97 & 1233397 & 1233545.2 & 125.82 & $\mathrm{~N} / \mathrm{A}$ & $\mathrm{N} / \mathrm{A}$ & $\mathrm{N} / \mathrm{A}$ & $\mathrm{N} / \mathrm{A}$ & $\mathrm{N} / \mathrm{A}$ \\
\hline as-22july06 & 2059 & 2059 & 0.05 & 2059 & 2059 & 1.67 & 2059 & 2059 & 2059 & 2059 & 19917 \\
\hline audikw1 & 10319 & 10432.7 & 2112.62 & 11092 & 11146.7 & 3411.03 & $\mathrm{~N} / \mathrm{A}$ & $\mathrm{N} / \mathrm{A}$ & 11864 & $\mathrm{~N} / \mathrm{A}$ & $\mathrm{N} / \mathrm{A}$ \\
\hline belgium & 1207728 & 1207843.7 & 26.78 & 1201674 & 1201683.8 & 20.68 & $\mathrm{~N} / \mathrm{A}$ & $\mathrm{N} / \mathrm{A}$ & N/A & $\mathrm{N} / \mathrm{A}$ & $\mathrm{N} / \mathrm{A}$ \\
\hline cage15 & 581885 & 582174.7 & 206.46 & 680979 & 684575.2 & 800.59 & $\mathrm{~N} / \mathrm{A}$ & $\mathrm{N} / \mathrm{A}$ & $\mathrm{N} / \mathrm{A}$ & $\mathrm{N} / \mathrm{A}$ & $\mathrm{N} / \mathrm{A}$ \\
\hline caidaRo*erLevel & 47922 & 47935.1 & 2363.89 & 47994 & 48002.8 & 3394.37 & 48361 & 48707.8 & 48422 & $\mathrm{~N} / \mathrm{A}$ & $\mathrm{N} / \mathrm{A}$ \\
\hline citatio*iteseer & 49494 & 49502.5 & 3148.56 & 49659 & 49696.8 & 3286.72 & 50488 & 51109 & 50434 & $\mathrm{~N} / \mathrm{A}$ & $\mathrm{N} / \mathrm{A}$ \\
\hline cnr-2000 & 24748 & 24751.6 & 3303.93 & 24780 & 24788.9 & 2374.51 & 25177 & 25190.5 & 24880 & $\mathrm{~N} / \mathrm{A}$ & $\mathrm{N} / \mathrm{A}$ \\
\hline coAutho*iteseer & 38104 & 38108 & 2088.81 & 38123 & 38128.9 & 2630.0 & 38203 & 38453.8 & 38265 & $\mathrm{~N} / \mathrm{A}$ & $\mathrm{N} / \mathrm{A}$ \\
\hline coAuthorsDBLP & 48774 & 48779.3 & 3101.11 & 48809 & 48820.5 & 2482.52 & 49103 & 49141.5 & 48963 & $\mathrm{~N} / \mathrm{A}$ & $\mathrm{N} / \mathrm{A}$ \\
\hline cond-mat-2005 & 5119 & 5119 & 21.46 & 5125 & 5127 & 345.32 & 5165 & 5169.3 & 5168 & 5175 & 33782 \\
\hline coPaper*iteseer & 34465 & 34494.6 & 3443.34 & 34781 & 34813.7 & 3575.36 & $\mathrm{~N} / \mathrm{A}$ & $\mathrm{N} / \mathrm{A}$ & $\mathrm{N} / \mathrm{A}$ & $\mathrm{N} / \mathrm{A}$ & $\mathrm{N} / \mathrm{A}$ \\
\hline coPapersDBLP & 45745 & 45773.6 & 3328.96 & 46392 & 46483.9 & 3546.35 & $\mathrm{~N} / \mathrm{A}$ & $\mathrm{N} / \mathrm{A}$ & $\mathrm{N} / \mathrm{A}$ & $\mathrm{N} / \mathrm{A}$ & $\mathrm{N} / \mathrm{A}$ \\
\hline ecology1 & 358317 & 360050.6 & 8.02 & 400020 & 403547.3 & 3599.42 & $\mathrm{~N} / \mathrm{A}$ & $\mathrm{N} / \mathrm{A}$ & $\mathrm{N} / \mathrm{A}$ & $\mathrm{N} / \mathrm{A}$ & $\mathrm{N} / \mathrm{A}$ \\
\hline eu-2005 & 34206 & 34217.7 & 3424.09 & 35146 & 35157.8 & 3320.62 & $\mathrm{~N} / \mathrm{A}$ & $\mathrm{N} / \mathrm{A}$ & $\mathrm{N} / \mathrm{A}$ & $\mathrm{N} / \mathrm{A}$ & $\mathrm{N} / \mathrm{A}$ \\
\hline G_n_pin_pout & 13481 & 13530.8 & 713.19 & 14280 & 14339.6 & 3361.24 & 15124 & 15154.4 & 15040 & 15351 & $\mathrm{~N} / \mathrm{A}$ \\
\hline in-2004 & 86863 & 86872.2 & 2944.51 & 86847 & 86876.4 & 2247.82 & $\mathrm{~N} / \mathrm{A}$ & $\mathrm{N} / \mathrm{A}$ & $\mathrm{N} / \mathrm{A}$ & $\mathrm{N} / \mathrm{A}$ & $\mathrm{N} / \mathrm{A}$ \\
\hline kron_g5*-logn 16 & 3886 & 3886 & 2.99 & 3886 & 3886.1 & 84.85 & 3888 & 3889.2 & 3887 & $\mathrm{~N} / \mathrm{A}$ & 53980 \\
\hline ldoor & 33436 & 33494.5 & 40.08 & 34086 & 34177.7 & 3571.83 & $\mathrm{~N} / \mathrm{A}$ & $\mathrm{N} / \mathrm{A}$ & $\mathrm{N} / \mathrm{A}$ & N/A & $\mathrm{N} / \mathrm{A}$ \\
\hline luxembourg & 101673 & 101695 & 3558.5 & 101552 & 101562.6 & 3577.45 & 102284 & 102305.8 & 101968 & $\mathrm{~N} / \mathrm{A}$ & 113387 \\
\hline prefere* achment & 8356 & 8359.1 & 3479.18 & 8544 & 8563.9 & 3240.6 & 9100 & 9116.3 & 9022 & 9155 & $\mathrm{~N} / \mathrm{A}$ \\
\hline rgg_n_2_17_s0 & 22518 & 22604.3 & 346.27 & 23146 & 23193.6 & 3570.98 & 23631 & 23662.3 & 23621 & $\mathrm{~N} / \mathrm{A}$ & $\mathrm{N} / \mathrm{A}$ \\
\hline rgg_n_2_19_s0 & 83791 & 83863 & 5.45 & 84063 & 84288.9 & 3585.14 & $\mathrm{~N} / \mathrm{A}$ & $\mathrm{N} / \mathrm{A}$ & $\mathrm{N} / \mathrm{A}$ & $\mathrm{N} / \mathrm{A}$ & $\mathrm{N} / \mathrm{A}$ \\
\hline rgg_n_2_20_s0 & 158867 & 158937.8 & 11.69 & 162289 & 163454.6 & 3541.92 & $\mathrm{~N} / \mathrm{A}$ & $\mathrm{N} / \mathrm{A}$ & $\mathrm{N} / \mathrm{A}$ & $\mathrm{N} / \mathrm{A}$ & $\mathrm{N} / \mathrm{A}$ \\
\hline rgg_n_2_21_s0 & 302163 & 302389.6 & 24.72 & 316284 & 318593.7 & 3599.47 & $\mathrm{~N} / \mathrm{A}$ & $\mathrm{N} / \mathrm{A}$ & $\mathrm{N} / \mathrm{A}$ & $\mathrm{N} / \mathrm{A}$ & $\mathrm{N} / \mathrm{A}$ \\
\hline rgg_n_2_22_s0 & $\mathbf{5 7 6 7 7 0}$ & 577023.8 & 48.17 & 620372 & 623562.6 & 3580.18 & $\mathrm{~N} / \mathrm{A}$ & $\mathrm{N} / \mathrm{A}$ & $\mathrm{N} / \mathrm{A}$ & $\mathrm{N} / \mathrm{A}$ & $\mathrm{N} / \mathrm{A}$ \\
\hline rgg_n_2_23_s0 & 1103096 & 1103648.3 & 110.17 & 1199201 & 1199450 & 140.13 & $\mathrm{~N} / \mathrm{A}$ & $\mathrm{N} / \mathrm{A}$ & $\mathrm{N} / \mathrm{A}$ & $\mathrm{N} / \mathrm{A}$ & $\mathrm{N} / \mathrm{A}$ \\
\hline rgg_n_2_24_s0 & 2115143 & 2115679.2 & 238.57 & 2325926 & 2326036.3 & 430.99 & $\mathrm{~N} / \mathrm{A}$ & $\mathrm{N} / \mathrm{A}$ & $\mathrm{N} / \mathrm{A}$ & $\mathrm{N} / \mathrm{A}$ & $\mathrm{N} / \mathrm{A}$ \\
\hline smallworld & 13785 & 13839.9 & 197.25 & 14355 & 14481 & 649.51 & 15354 & 15374.6 & 15609 & 15646 & $\mathrm{~N} / \mathrm{A}$ \\
\hline uk-2002 & 1160524 & 1160612.8 & 1708.24 & 1184688 & 1184715.3 & 288.93 & $\mathrm{~N} / \mathrm{A}$ & $\mathrm{N} / \mathrm{A}$ & $\mathrm{N} / \mathrm{A}$ & $\mathrm{N} / \mathrm{A}$ & $\mathrm{N} / \mathrm{A}$ \\
\hline wave & 15744 & 15861.3 & 226.38 & 16285 & 16365.8 & 3582.3 & 17504 & 17547.8 & 17792 & $\mathrm{~N} / \mathrm{A}$ & $\mathrm{N} / \mathrm{A}$ \\
\hline
\end{tabular}

Table 4: Results of FastCDS, NuCDS, MSLS, ACO-efficient, ACO-RVNS and RNS-TS on DIMACS10 and SNAP benchmarks.

Two reasons accounting for why competitors cannot output feasible solutions on some instances are as follows: 1) The construction processes of MSLS, ACO and ACO-efficient have a complexity of $\mathrm{O}\left(|V|^{2}\right)$. They are essential parts of multi-restart and ACO framework, so we reserve them; 2) The complexity of each step is $\mathrm{O}\left(|V|^{2}\right)$ in RNS, often trapping in the first iteration. Because $|V|$ of some instances reaches to $10^{7}$, competitors fail to output a feasible solution. 
ZHANG, Li, CAI, \& WANG

\begin{tabular}{|c|c|c|c|c|c|c|c|c|c|c|}
\hline \multirow{2}{*}{ Instance } & \multicolumn{3}{|l|}{ FastCDS } & \multicolumn{3}{|l|}{$\mathrm{NuCDS}$} & \multirow{2}{*}{$\frac{\text { MSLS }}{\min }$} & \multirow{2}{*}{$\frac{\mathrm{ACO}_{e}}{\min }$} & \multirow{2}{*}{$\frac{\mathrm{ACO}}{\min }$} & \multirow{2}{*}{$\begin{array}{l}\text { RNS } \\
\min \end{array}$} \\
\hline & $\min$ & avg & time & $\min$ & avg & time & & & & \\
\hline bn-huma*on_1-bg & 4212 & 4218.3 & 3337.39 & 4417 & 4511.6 & 3598.07 & 4683 & 4692 & $\mathrm{~N} / \mathrm{A}$ & $\mathrm{N} / \mathrm{A}$ \\
\hline bn-huma*on_2-bg & 3345 & 3353.4 & 3595.88 & 3454 & 3487.5 & 3462.56 & 3836 & 3704 & $\mathrm{~N} / \mathrm{A}$ & $\mathrm{N} / \mathrm{A}$ \\
\hline ca-coau*rs-dblp & 45745 & 45773.6 & 3099.34 & 46392 & 46493.6 & 3396.21 & $\mathrm{~N} / \mathrm{A}$ & $\mathrm{N} / \mathrm{A}$ & $\mathrm{N} / \mathrm{A}$ & $\mathrm{N} / \mathrm{A}$ \\
\hline ca-dblp-2012 & 50997 & 51003.4 & 1399.86 & 51015 & 51029.6 & 2479.22 & 51072 & 51193 & $\mathrm{~N} / \mathrm{A}$ & 316258 \\
\hline ca-holl*od-2009 & 51503 & 51599.9 & 3598.88 & 53062 & 53259.8 & 3589.33 & $\mathrm{~N} / \mathrm{A}$ & $\mathrm{N} / \mathrm{A}$ & $\mathrm{N} / \mathrm{A}$ & $\mathrm{N} / \mathrm{A}$ \\
\hline 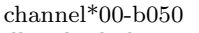 & 580856 & 581204.2 & 76.14 & 653869 & 654154 & 879.59 & $\mathrm{~N} / \mathrm{A}$ & $\mathrm{N} / \mathrm{A}$ & $\mathrm{N} / \mathrm{A}$ & $\mathrm{N} / \mathrm{A}$ \\
\hline dbpedia-link & 1533532 & 1533536.7 & 3520.33 & $\mathrm{~N} / \mathrm{A}$ & $\mathrm{N} / \mathrm{A}$ & $\mathrm{N} / \mathrm{A}$ & $\mathrm{N} / \mathrm{A}$ & $\mathrm{N} / \mathrm{A}$ & $\mathrm{N} / \mathrm{A}$ & $\mathrm{N} / \mathrm{A}$ \\
\hline delaunay_n22 & 1125248 & 1125473 & 166.83 & 1188266 & 1188358.1 & 102.31 & $\mathrm{~N} / \mathrm{A}$ & $\mathrm{N} / \mathrm{A}$ & $\mathrm{N} / \mathrm{A}$ & $\mathrm{N} / \mathrm{A}$ \\
\hline delaunay_n23 & 2250110 & 2250432.9 & 689.43 & 2368743 & 2376193.4 & 2900.29 & $\mathrm{~N} / \mathrm{A}$ & $\mathrm{N} / \mathrm{A}$ & $\mathrm{N} / \mathrm{A}$ & $\mathrm{N} / \mathrm{A}$ \\
\hline delaunay_n24 & 4507091 & 4507747.8 & 188.48 & 4866995 & 4867078.2 & 158.46 & $\mathrm{~N} / \mathrm{A}$ & $\mathrm{N} / \mathrm{A}$ & $\mathrm{N} / \mathrm{A}$ & $\mathrm{N} / \mathrm{A}$ \\
\hline friendster & 649542 & 649555.4 & 1056.58 & 659802 & 659919.8 & 1170.39 & $\mathrm{~N} / \mathrm{A}$ & $\mathrm{N} / \mathrm{A}$ & $\mathrm{N} / \mathrm{A}$ & $\mathrm{N} / \mathrm{A}$ \\
\hline hugebub*s-00020 & 11431627 & 11475735.4 & 3059.0 & 12438068 & 12554344.1 & 3234.79 & $\mathrm{~N} / \mathrm{A}$ & $\mathrm{N} / \mathrm{A}$ & $\mathrm{N} / \mathrm{A}$ & $\mathrm{N} / \mathrm{A}$ \\
\hline hugetrace-00010 & 6510298 & 6510786.9 & 284.69 & 7014767 & 7119364.1 & 2857.97 & $\mathrm{~N} / \mathrm{A}$ & $\mathrm{N} / \mathrm{A}$ & $\mathrm{N} / \mathrm{A}$ & $\mathrm{N} / \mathrm{A}$ \\
\hline hugetrace- 00020 & 8631122 & 8633275.2 & 1717.65 & 9493668 & 9514712.4 & 356.83 & $\mathrm{~N} / \mathrm{A}$ & $\mathrm{N} / \mathrm{A}$ & $\mathrm{N} / \mathrm{A}$ & $\mathrm{N} / \mathrm{A}$ \\
\hline inf-europe_osm & 43987750 & 43992418.2 & 3599.73 & 43785238 & 43785337 & 3543.52 & $\mathrm{~N} / \mathrm{A}$ & $\mathrm{N} / \mathrm{A}$ & $\mathrm{N} / \mathrm{A}$ & $\mathrm{N} / \mathrm{A}$ \\
\hline inf-germany_osm & 9547995 & 9549133.5 & 3590.4 & 9497412 & 9497590.6 & 2771.98 & $\mathrm{~N} / \mathrm{A}$ & $\mathrm{N} / \mathrm{A}$ & $\mathrm{N} / \mathrm{A}$ & $\mathrm{N} / \mathrm{A}$ \\
\hline inf-roadNet-CA & 969870 & 970396.2 & 35.4 & 1015787 & 1015838.3 & 13.67 & $\mathrm{~N} / \mathrm{A}$ & $\mathrm{N} / \mathrm{A}$ & $\mathrm{N} / \mathrm{A}$ & $\mathrm{N} / \mathrm{A}$ \\
\hline inf-roadNet-PA & 535031 & 535677.6 & 17.75 & 539827 & 546456.7 & 3599.86 & $\mathrm{~N} / \mathrm{A}$ & $\mathrm{N} / \mathrm{A}$ & $\mathrm{N} / \mathrm{A}$ & $\mathrm{N} / \mathrm{A}$ \\
\hline inf-road-usa & 14250176 & 14256927.6 & 3594.54 & 14395692 & 14395784.3 & 223.37 & $\mathrm{~N} / \mathrm{A}$ & $\mathrm{N} / \mathrm{A}$ & $\mathrm{N} / \mathrm{A}$ & $\mathrm{N} / \mathrm{A}$ \\
\hline rec-dating & 11739 & 11740.5 & 3312.85 & 11745 & 11747.1 & 3306.1 & 11748 & 11754 & $\mathrm{~N} / \mathrm{A}$ & $\mathrm{N} / \mathrm{A}$ \\
\hline rec-epinions & 9067 & 9067.3 & 2013.69 & 9080 & 9084 & 2762.39 & $\mathrm{~N} / \mathrm{A}$ & 9100 & $\mathrm{~N} / \mathrm{A}$ & $\mathrm{N} / \mathrm{A}$ \\
\hline rec-lib*eti-dir & 12956 & 12958.9 & 3354.25 & 12977 & 13001.4 & 3497.2 & $\mathrm{~N} / \mathrm{A}$ & 13031 & $\mathrm{~N} / \mathrm{A}$ & $\mathrm{N} / \mathrm{A}$ \\
\hline rgg_n_2_23_s0 & 1103096 & 1103648.3 & 110.17 & 1199201 & 1199450 & 140.13 & $\mathrm{~N} / \mathrm{A}$ & $\mathrm{N} / \mathrm{A}$ & $\mathrm{N} / \mathrm{A}$ & $\mathrm{N} / \mathrm{A}$ \\
\hline rgg_n_2_24_s0 & 2115143 & 2115679.2 & 238.57 & 2325926 & 2326036.3 & 430.99 & $\mathrm{~N} / \mathrm{A}$ & $\mathrm{N} / \mathrm{A}$ & $\mathrm{N} / \mathrm{A}$ & $\mathrm{N} / \mathrm{A}$ \\
\hline rt-retw*t-crawl & 82910 & 82916.1 & 3446.08 & 83117 & 83120 & 3338.63 & $\mathrm{~N} / \mathrm{A}$ & $\mathrm{N} / \mathrm{A}$ & $\mathrm{N} / \mathrm{A}$ & $\mathrm{N} / \mathrm{A}$ \\
\hline sc-ldoor & 33384 & 33447.5 & 40.73 & 34151 & 34205.4 & 3531.43 & $\mathrm{~N} / \mathrm{A}$ & $\mathrm{N} / \mathrm{A}$ & $\mathrm{N} / \mathrm{A}$ & $\mathrm{N} / \mathrm{A}$ \\
\hline sc-msdoor & 14673 & 14728.1 & 462.77 & 15071 & 15101.8 & 3432.13 & 15279 & 15391 & $\mathrm{~N} / \mathrm{A}$ & $\mathrm{N} / \mathrm{A}$ \\
\hline sc-pwtk & 8471 & 8512.7 & 111.41 & 8757 & 8802.1 & 3451.43 & 8819 & 8931 & $\mathrm{~N} / \mathrm{A}$ & $\mathrm{N} / \mathrm{A}$ \\
\hline sc-rel9 & 120681 & 121304.9 & 3546.2 & 124320 & 125264.9 & 3599.17 & $\mathrm{~N} / \mathrm{A}$ & $\mathrm{N} / \mathrm{A}$ & $\mathrm{N} / \mathrm{A}$ & $\mathrm{N} / \mathrm{A}$ \\
\hline sc-shipsec1 & 10631 & 10729 & 607.79 & 10937 & 10998.8 & 2380.66 & 11791 & 11954 & $\mathrm{~N} / \mathrm{A}$ & 13766 \\
\hline sc-shipsec5 & 13283 & 13694.4 & 3042.98 & 13811 & 13921.5 & 2143.77 & 14839 & 15082 & $\mathrm{~N} / \mathrm{A}$ & 176869 \\
\hline soc-buzznet & 128 & 128 & 9.9 & 128 & 128 & 38.79 & 128 & 128 & $\mathrm{~N} / \mathrm{A}$ & 99847 \\
\hline soc-delicious & 57662 & 57666.9 & 1106.46 & 57685 & 57688.3 & 3037.08 & $\mathrm{~N} / \mathrm{A}$ & $\mathrm{N} / \mathrm{A}$ & $\mathrm{N} / \mathrm{A}$ & 535742 \\
\hline soc-digg & 70626 & 70637.9 & 3596.03 & 70654 & 70664.9 & 3596.29 & $\mathrm{~N} / \mathrm{A}$ & $\mathrm{N} / \mathrm{A}$ & $\mathrm{N} / \mathrm{A}$ & N/A \\
\hline soc-dogster & 27216 & 27218.4 & 3340.64 & 27284 & 27291.1 & 2614.78 & 27359 & 27391 & $\mathrm{~N} / \mathrm{A}$ & $\mathrm{N} / \mathrm{A}$ \\
\hline socfb-A-anon & 204650 & 204656.5 & 2767.83 & 206186 & 206809.1 & 3499.2 & $\mathrm{~N} / \mathrm{A}$ & $\mathrm{N} / \mathrm{A}$ & $\mathrm{N} / \mathrm{A}$ & $\mathrm{N} / \mathrm{A}$ \\
\hline socfb-B-anon & 190246 & 190252.2 & 124.78 & 192419 & 193223.6 & 2588.29 & $\mathrm{~N} / \mathrm{A}$ & $\mathrm{N} / \mathrm{A}$ & $\mathrm{N} / \mathrm{A}$ & $\mathrm{N} / \mathrm{A}$ \\
\hline socfb-uci-uni & 1237712 & 1237862.4 & 3453.03 & 1542814 & 16666186.3 & 3033.06 & $\mathrm{~N} / \mathrm{A}$ & $\mathrm{N} / \mathrm{A}$ & $\mathrm{N} / \mathrm{A}$ & $\mathrm{N} / \mathrm{A}$ \\
\hline soc-flickr & 105687 & 105694.6 & 23.83 & 105659 & 105664.5 & 3210.59 & $\mathrm{~N} / \mathrm{A}$ & $\mathrm{N} / \mathrm{A}$ & $\mathrm{N} / \mathrm{A}$ & 513557 \\
\hline soc-flickr-und & 296567 & 296575.6 & 169.68 & 297000 & 297280 & 3577.6 & $\mathrm{~N} / \mathrm{A}$ & $\mathrm{N} / \mathrm{A}$ & $\mathrm{N} / \mathrm{A}$ & $\mathrm{N} / \mathrm{A}$ \\
\hline soc-flixster & 91544 & 91544.2 & 1807.06 & 91545 & 91545.6 & 47.51 & $\mathrm{~N} / \mathrm{A}$ & $\mathrm{N} / \mathrm{A}$ & $\mathrm{N} / \mathrm{A}$ & $\mathrm{N} / \mathrm{A}$ \\
\hline soc-FourSquare & 60980 & 60981.7 & 3502.39 & 60979 & 60982 & 2853.2 & $\mathrm{~N} / \mathrm{A}$ & $\mathrm{N} / \mathrm{A}$ & $\mathrm{N} / \mathrm{A}$ & 638426 \\
\hline soc-lastfm & 67424 & 67424 & 5.88 & 67429 & 67429 & 558.08 & $\mathrm{~N} / \mathrm{A}$ & $\mathrm{N} / \mathrm{A}$ & $\mathrm{N} / \mathrm{A}$ & $\mathrm{N} / \mathrm{A}$ \\
\hline soc-livejournal & 842865 & 842897.3 & 1295.58 & 854550 & 854557.4 & 152.82 & $\mathrm{~N} / \mathrm{A}$ & $\mathrm{N} / \mathrm{A}$ & $\mathrm{N} / \mathrm{A}$ & $\mathrm{N} / \mathrm{A}$ \\
\hline soc-liv*-groups & 1101690 & 1101710.4 & 3595.97 & $\mathrm{~N} / \mathrm{A}$ & $\mathrm{N} / \mathrm{A}$ & $\mathrm{N} / \mathrm{A}$ & $\mathrm{N} / \mathrm{A}$ & $\mathrm{N} / \mathrm{A}$ & $\mathrm{N} / \mathrm{A}$ & $\mathrm{N} / \mathrm{A}$ \\
\hline soc-LiveMocha & 1424 & 1424 & 85.51 & 1425 & 1426.9 & 2979.29 & 1430 & 1454 & 1476 & 103049 \\
\hline soc-ljo*al-2008 & 1061607 & 1061692.4 & 1962.46 & 1071978 & 1071990.7 & 347.33 & $\mathrm{~N} / \mathrm{A}$ & $\mathrm{N} / \mathrm{A}$ & $\mathrm{N} / \mathrm{A}$ & $\mathrm{N} / \mathrm{A}$ \\
\hline soc-orkut-dir & 94975 & 95380.6 & 3576.63 & 100708 & 101023.3 & 3570.36 & $\mathrm{~N} / \mathrm{A}$ & $\mathrm{N} / \mathrm{A}$ & $\mathrm{N} / \mathrm{A}$ & $\mathrm{N} / \mathrm{A}$ \\
\hline soc-orkut & 113264 & 114206.2 & 3597.64 & 120762 & 120854.1 & 3583.99 & $\mathrm{~N} / \mathrm{A}$ & $\mathrm{N} / \mathrm{A}$ & $\mathrm{N} / \mathrm{A}$ & $\mathrm{N} / \mathrm{A}$ \\
\hline soc-pokec & 214958 & 215137.4 & 3594.25 & 221800 & 222244.3 & 3592.73 & $\mathrm{~N} / \mathrm{A}$ & $\mathrm{N} / \mathrm{A}$ & $\mathrm{N} / \mathrm{A}$ & $\mathrm{N} / \mathrm{A}$ \\
\hline soc-sinaweibo & 201399 & 201399 & 510.02 & $\mathrm{~N} / \mathrm{A}$ & $\mathrm{N} / \mathrm{A}$ & $\mathrm{N} / \mathrm{A}$ & $\mathrm{N} / \mathrm{A}$ & $\mathrm{N} / \mathrm{A}$ & $\mathrm{N} / \mathrm{A}$ & $\mathrm{N} / \mathrm{A}$ \\
\hline soc-twi*r-higgs & 14961 & 14965.9 & 2689.14 & 15164 & 15183.6 & 3378.36 & 15335 & 15375 & $\mathrm{~N} / \mathrm{A}$ & $\mathrm{N} / \mathrm{A}$ \\
\hline soc-youtube & 101716 & 101723.4 & 3235.94 & 101657 & 101663.7 & 3584.01 & $\mathrm{~N} / \mathrm{A}$ & $\mathrm{N} / \mathrm{A}$ & $\mathrm{N} / \mathrm{A}$ & 495497 \\
\hline soc-you*be-snap & 235634 & 235641.4 & 85.2 & 235575 & 235598.8 & 2952.14 & $\mathrm{~N} / \mathrm{A}$ & $\mathrm{N} / \mathrm{A}$ & $\mathrm{N} / \mathrm{A}$ & $\mathrm{N} / \mathrm{A}$ \\
\hline tech-as-skitter & 197800 & 197843.5 & 3463.31 & 199864 & 200270.5 & 3598.07 & $\mathrm{~N} / \mathrm{A}$ & $\mathrm{N} / \mathrm{A}$ & $\mathrm{N} / \mathrm{A}$ & $\mathrm{N} / \mathrm{A}$ \\
\hline tech-ip & 153 & 153.6 & 2615.95 & 153 & 153.9 & 2790.7 & $\mathrm{~N} / \mathrm{A}$ & 170 & $\mathrm{~N} / \mathrm{A}$ & $\mathrm{N} / \mathrm{A}$ \\
\hline twitter_mpi & 567536 & 567543 & 1313.45 & $\mathrm{~N} / \mathrm{A}$ & $\mathrm{N} / \mathrm{A}$ & $\mathrm{N} / \mathrm{A}$ & $\mathrm{N} / \mathrm{A}$ & $\mathrm{N} / \mathrm{A}$ & $\mathrm{N} / \mathrm{A}$ & $\mathrm{N} / \mathrm{A}$ \\
\hline web-arabic- 2005 & 20646 & 20648.6 & 410.96 & 20663 & 20670.9 & 676.52 & 20737 & 20730 & $\mathrm{~N} / \mathrm{A}$ & 162433 \\
\hline web-baidu-baike & 273145 & 273159.2 & 428.08 & 273824 & 274001.6 & 3590.9 & $\mathrm{~N} / \mathrm{A}$ & $\mathrm{N} / \mathrm{A}$ & $\mathrm{N} / \mathrm{A}$ & $\mathrm{N} / \mathrm{A}$ \\
\hline web-it-2004 & 34548 & 34548.1 & 7.15 & 34549 & 34549.3 & 132.54 & 34551 & 34557 & $\mathrm{~N} / \mathrm{A}$ & $\mathrm{N} / \mathrm{A}$ \\
\hline web-uk-2005 & 1728 & 1728 & 10.62 & 1728 & 1728 & 1.62 & 1728 & 1728 & 1728 & $\mathrm{~N} / \mathrm{A}$ \\
\hline web-wik*dia2009 & 394301 & 394339.1 & 288.63 & 396303 & 397633.5 & 3598.67 & $\mathrm{~N} / \mathrm{A}$ & $\mathrm{N} / \mathrm{A}$ & $\mathrm{N} / \mathrm{A}$ & $\mathrm{N} / \mathrm{A}$ \\
\hline web-wik*-growth & 117956 & 118043.8 & 3591.71 & 118964 & 118976.4 & 1243.04 & $\mathrm{~N} / \mathrm{A}$ & $\mathrm{N} / \mathrm{A}$ & $\mathrm{N} / \mathrm{A}$ & $\mathrm{N} / \mathrm{A}$ \\
\hline web-wik*ia_link & 190180 & 190191.4 & 1050.96 & 190982 & 191063.83 & 2848.3 & $\mathrm{~N} / \mathrm{A}$ & $\mathrm{N} / \mathrm{A}$ & $\mathrm{N} / \mathrm{A}$ & $\mathrm{N} / \mathrm{A}$ \\
\hline wikiped*link_en & 212242 & 212243.1 & 150.98 & 212242 & 212257.7 & 2807.97 & $\mathrm{~N} / \mathrm{A}$ & $\mathrm{N} / \mathrm{A}$ & $\mathrm{N} / \mathrm{A}$ & $\mathrm{N} / \mathrm{A}$ \\
\hline
\end{tabular}

Table 5: Results of FastCDS, NuCDS, MSLS, ACO-efficient, ACO-RVNS and RNS-TS on NDR benchmarks. 


\subsection{Effectiveness of Proposed Strategies}

As shown in Table 6, six modified versions of FastCDS are proposed to verify the effectiveness of each strategy of our algorithm on massive graphs. We compare FastCDS with FastCDS 1 to show the effectiveness of chrono-safety, with FastCDS 2 and FastCDS 3 to show the effectiveness of HDC+. Moreover, we compare FastCDS with FastCDS 4 to show the effectiveness of the novel age and safety combination method, and with FastCDS ${ }_{5}$ to show the effectiveness of reconstruction method in $\mathrm{HDC}+$, and with FastCDS 6 , where all vertices are in $V_{\text {cand }}$, to show the effectiveness brought by the partition of vertices (i.e., $V_{\text {in }}, V_{\text {out }}$ and $\left.V_{\text {cand }}\right)$. Meanwhile, when compare with FastCDS 2 , which only uses SUB, FastCDS improves the average best solution by 9753.77 on massive graphs. The results of comparisons are shown in Table 7.

In addition, in our previous work, The HDC method of NuCDS improves the average best solution by $17.285 \%$ on massive graphs when comparing with its modified version which only uses SUB, while for FastCDS the number is $0.928 \%$ when compared with FastCDS 2 , mainly because the initial solution of FastCDS is much better than that of NuCDS, which confirms the effectiveness of 1hopReason.

\begin{tabular}{lllllll}
\hline & FastCDS $_{1}$ & FastCDS $_{2}$ & FastCDS $_{3}$ & FastCDS $_{4}$ & FastCDS $_{5}$ & FastCDS $_{6}$ \\
\hline HDC + & + & - & - & + & - & + \\
HDC & - & - & - & - & + & - \\
only-SUB & - & + & - & - & - & - \\
only-TBC & - & - & + & - & - & - \\
safety & - & - & - & + & - & - \\
chrono-safety & - & + & + & - & + & + \\
Partition & + & + & + & + & + & - \\
\hline
\end{tabular}

Table 6: Six modified versions of FastCDS, where "+" indicates that the version uses the corresponding strategy while "-" means not.

\begin{tabular}{|c|c|c|c|c|c|c|c|}
\hline Benchmark & & vs.FastCDS 1 & vs.FastCDS 2 & vs.FastCDS 3 & vs.FastCDS 4 & vs.FastCDS 5 & vs.FastCDS 6 \\
\hline \multirow{2}{*}{ DIMACS10(22) } & \#Better & 8 & 11 & 6 & 11 & 19 & 7 \\
\hline & \#Worse & 2 & 2 & 1 & 3 & 0 & 5 \\
\hline \multirow{2}{*}{$\operatorname{SNAP}(31)$} & \#Better & 22 & 26 & 7 & 14 & 27 & 18 \\
\hline & \#Worse & 6 & 2 & 7 & 14 & 2 & 7 \\
\hline \multirow{2}{*}{ NDR(65) } & \#Better & 32 & 58 & 24 & 43 & 52 & 42 \\
\hline & \#Worse & 21 & 0 & 10 & 13 & 4 & 9 \\
\hline
\end{tabular}

Table 7: Comparing FastCDS with 6 modified versions on massive graphs. \#Better and \#Worse represent respectively the number of instances where FastCDS achieves better and worse results.

\subsection{Further Comparison of Different Construction Algorithms}

In this subsection, we carry out experiments to compare our construction algorithm, 1hopReason, with 3 construction algorithms, namely 1 hop*, 2 hop* and 1hopNoReason. Specifically, 1hop* and 2hop* denote the improved 1-hop and 2-hop local greedy algorithms (Khuller \& Yang, 2019) respectively, which are the state-of-the-art local information greedy 


\begin{tabular}{|c|c|c|c|c|c|c|c|c|c|c|c|c|}
\hline \multirow{2}{*}{ Instance } & \multirow{2}{*}{$|V|$} & \multirow{2}{*}{$|E|$} & \multirow[b]{2}{*}{$\left|V_{i n}\right|$} & \multirow[b]{2}{*}{$\left|V_{\text {out }}\right|$} & \multicolumn{3}{|c|}{ 1hopReason } & \multicolumn{3}{|c|}{ 1hopNoReason } & \multirow{2}{*}{$\frac{1 \text { hop* }}{\min }$} & \multirow{2}{*}{$\frac{2 \mathrm{hop}^{*}}{\min }$} \\
\hline & & & & & $\min$ & avg & time & $\min$ & avg & time & & \\
\hline Amazon0302 & 262111 & 899792 & 7371 & 18283 & 48321 & 48378.4 & 1.44 & 48818 & 48843.5 & 0.98 & 61616 & 67775 \\
\hline Amazon0312 & 400727 & 2349869 & 17981 & 37365 & 54686 & 54722.1 & 2.88 & 56034 & 56081.1 & 2.67 & 72796 & 77667 \\
\hline Amazon0505 & 410236 & 2439437 & 19307 & 40088 & 56568 & 56624.8 & 2.95 & 57966 & 58022.5 & 2.82 & 75152 & 77311 \\
\hline Amazon0601 & 403364 & 2443311 & 12541 & 32057 & 50682 & 50712 & 3.45 & 51773 & 51788.2 & 2.69 & 67116 & 72354 \\
\hline Cit-HepPh & 34401 & 420784 & 1169 & 1995 & 3433 & 3444.6 & 0.41 & 3498 & 3509.6 & 0.4 & 4510 & 3949 \\
\hline Cit-HepTh & 27400 & 352021 & 1316 & 2039 & 3349 & 3358.1 & 0.34 & 3426 & 3431.3 & 0.34 & 4225 & 3952 \\
\hline cit-Patents & 3764117 & 16511740 & 407793 & 709062 & 700525 & 700605 & 31.13 & 723239 & 723345.2 & 24.42 & 917079 & 924107 \\
\hline Email-EuAll & 224832 & 339925 & 2223 & 202193 & 2371 & 2371.8 & 0.39 & 2373 & 2374.4 & 0.36 & 3175 & 4641 \\
\hline p2p-Gnutella04 & 10876 & 39994 & 1757 & 2484 & 2295 & 2297.4 & 0.04 & 2416 & 2429 & 0.04 & 3321 & 2653 \\
\hline p2p-Gnutella24 & 26498 & 65359 & 5147 & 10994 & 5473 & 5475.1 & 0.07 & 5585 & 5592.9 & 0.07 & 8005 & 6149 \\
\hline p2p-Gnutella25 & 22663 & 54693 & 4273 & 9324 & 4561 & 4563.3 & 0.06 & 4660 & 4673.8 & 0.06 & 6670 & 5063 \\
\hline p2p-Gnutella30 & 36646 & 88303 & 6888 & 16499 & 7231 & 7231.9 & 0.1 & 7374 & 7380.4 & 0.09 & 10513 & 8429 \\
\hline p2p-Gnutella31 & 62561 & 147878 & 12252 & 28806 & 12676 & 12677.8 & 0.16 & 12853 & 12880.9 & 0.16 & 18210 & 14359 \\
\hline Slashdot0811 & 77360 & 469180 & 13832 & 30164 & 14998 & 14999.3 & 0.48 & 15029 & 15032 & 0.47 & 20129 & 15254 \\
\hline Slashdot0902 & 82168 & 504230 & 14363 & 30855 & 16175 & 16178 & 0.54 & 16220 & 16223.1 & 0.49 & 21230 & 16530 \\
\hline soc-Epinions1 & 75877 & 405739 & 15936 & 41046 & 16677 & 16677.2 & 0.4 & 16703 & 16705.7 & 0.39 & 22109 & 16883 \\
\hline web-BerkStan & 654782 & 6581871 & 12977 & 145874 & 31373 & 31396.7 & 19.4 & 31501 & 31519.7 & 6.34 & 37170 & 37065 \\
\hline web-Google & 855802 & 4291352 & 59794 & 333051 & 88026 & 88049.6 & 5.7 & 89048 & 89078.1 & 4.43 & 112411 & 107650 \\
\hline web-NotreDame & 325729 & 1090108 & 21780 & 183085 & 25630 & 25654 & 1.33 & 25744 & 25762.7 & 1.1 & 33005 & 29004 \\
\hline web-Stanford & 255265 & 1941926 & 5458 & 50670 & 11777 & 11792 & 2.94 & 11818 & 11831.7 & 1.86 & 14063 & 13813 \\
\hline WikiTalk & 2388953 & 4656682 & 34281 & 1842442 & 35042 & 35042 & 7.16 & 35042 & 35042 & 5.0 & 47596 & 40781 \\
\hline Wiki-Vote & 7066 & 100736 & 1030 & 2471 & 1101 & 1101.3 & 0.09 & 1104 & 1104.4 & 0.09 & 1519 & 1106 \\
\hline $333 \mathrm{SP}$ & 3712815 & 11108633 & 0 & 8 & 1202378 & 1202555.8 & 13.96 & 1202112 & 1202539.5 & 12.52 & 1367950 & 1390253 \\
\hline as-22july06 & 22963 & 48436 & 1870 & 11927 & 2059 & 2060.2 & 0.06 & 2062 & 2063.1 & 0.05 & 2859 & 2346 \\
\hline audikw1 & 943695 & 38354076 & 0 & 2658 & 12147 & 12186 & 37.9 & 12159 & 12189.6 & 35.58 & 15988 & 19373 \\
\hline belgium & 1441295 & 1549970 & 211952 & 52575 & 1216936 & 1217055.1 & 3.09 & 1217159 & 1217234.9 & 2.1 & 1260193 & 1206836 \\
\hline cage15 & 5154859 & 47022346 & 0 & 128 & 586757 & 586947.2 & 59.72 & 586749 & 586959.7 & 52.16 & 695138 & 1154163 \\
\hline caidaRo*erLevel & 190914 & 607610 & 30228 & 52004 & 48669 & 48682.7 & 0.73 & 48977 & 48997.6 & 0.68 & 61017 & 53648 \\
\hline citatio*iteseer & 268495 & 1156647 & 31071 & 49457 & 50491 & 50502.9 & 1.36 & 51237 & 51279.6 & 1.35 & 65702 & 61691 \\
\hline cnr-2000 & 325557 & 2738969 & 19545 & 126537 & 25003 & 25019.8 & 4.64 & 25174 & 25184.1 & 2.56 & 31275 & 36180 \\
\hline coAutho*iteseer & 227320 & 814134 & 29867 & 145237 & 38298 & 38306 & 0.91 & 38743 & 38752.7 & 0.93 & 50459 & 40728 \\
\hline coAuthorsDBLP & 299067 & 977676 & 38269 & 184302 & 48983 & 48989.9 & 1.46 & 49426 & 49436.7 & 1.12 & 66052 & 51728 \\
\hline cond-mat-2005 & 36458 & 171734 & 3049 & 19107 & 5200 & 5203.3 & 0.17 & 5262 & 5267.1 & 0.17 & 7033 & 5543 \\
\hline coPaper*iteseer & 434102 & 16036720 & 10951 & 180347 & 36014 & 36027 & 15.54 & 36326 & 36341.1 & 15.44 & 45830 & 39529 \\
\hline coPapersDBLP & 540486 & 15245729 & 16301 & 231168 & 48218 & 48229.1 & 14.9 & 48770 & 48785.3 & 14.22 & 61921 & 53475 \\
\hline ecology 1 & 1000000 & 1998000 & 0 & 0 & 359585 & 361515.6 & 2.43 & 359585 & 361515.6 & 2.24 & 454883 & 424932 \\
\hline eu-2005 & 862664 & 16138468 & 24873 & 126473 & 34782 & 34797.7 & 23.7 & 34911 & 34932.6 & 15.21 & 43921 & 52841 \\
\hline G_n_pin_pout & 99995 & 501198 & 53 & 54 & 15414 & 15437.6 & 0.68 & 15404 & 15430.8 & 0.51 & 19280 & 24187 \\
\hline in-2004 & 1353703 & 13126172 & 66448 & 521664 & 87562 & 87576.6 & 19.21 & 88044 & 88054.9 & 12.56 & 109688 & 112797 \\
\hline kron_g5*-logn 16 & 55319 & 2456070 & 3251 & 8604 & 3888 & 3889.2 & 2.45 & 3897 & 3899.4 & 2.3 & 5223 & 3896 \\
\hline ldoor & 952203 & 22785136 & 0 & 0 & 34665 & 34713.8 & 22.32 & 34665 & 34713.8 & 21.15 & 41290 & 47529 \\
\hline luxembourg & 114599 & 119666 & 22426 & 3948 & 102257 & 102287.6 & 0.15 & 102248 & 102302.7 & 0.14 & 105146 & 101870 \\
\hline prefere*achment & 100000 & 499985 & 0 & 0 & 9171 & 9196.3 & 0.54 & 9171 & 9196.3 & 0.51 & 12441 & 10122 \\
\hline rgg_n_2_17_s0 & 131067 & 728750 & 25 & 704 & 23657 & 23709.3 & 0.77 & 23688 & 23716.6 & 0.72 & 28144 & 28649 \\
\hline rgg_n_2_19_s0 & 524280 & 3269760 & 47 & 1274 & 84655 & 84700.7 & 3.73 & 84639 & 84672.9 & 3.48 & 100599 & 105553 \\
\hline rgg_n_2_20_s0 & 1048572 & 6891617 & 54 & 1723 & 160652 & 160803.3 & 7.34 & 160688 & 160801.9 & 6.99 & 190501 & 201899 \\
\hline rgg_n_2_21_s0 & 2097142 & 14487992 & 54 & 2267 & 306238 & 306395.4 & 16.22 & 306182 & 306444.4 & 15.83 & 363352 & 389032 \\
\hline rgg_n_2_22_s0 & 4194299 & 30359197 & 65 & 2908 & 585191 & 585364.1 & 32.47 & 585139 & 585434.4 & 34.46 & 694961 & 751498 \\
\hline rgg_n_2_23_s0 & 8388601 & 63501390 & 53 & 246 & 1120430 & 1120729.8 & 70.24 & 1120661 & 1120823.1 & 68.06 & 1329443 & 1449467 \\
\hline rgg_n_2_24_s0 & 16777215 & 132557200 & 68 & 232 & 2150059 & 2150502.1 & 144.87 & 2150017 & 2150374.3 & 142.7 & 2550995 & 2806078 \\
\hline smallworld & 100000 & 499998 & 0 & 0 & 15771 & 15821.1 & 0.57 & 15771 & 15821.1 & 0.51 & 19543 & 26632 \\
\hline uk-2002 & 18459128 & 261556721 & 773160 & 3049470 & 1175517 & 1175713.3 & 263.63 & 1185305 & 1185487.3 & 253.68 & 1469643 & 1568781 \\
\hline wave & 156317 & 1059331 & 0 & 13 & 18060 & 18149 & 1.25 & 18118 & 18171.3 & 1.07 & 23233 & 29430 \\
\hline
\end{tabular}

Table 8: Results of 1hopReason, 1hopNoReason, 1hop*, 2hop* on DIMACS10 and SNAP benchmarks.

algorithms with the best approximation ratio as far as we know. 1hopNoReason is the modified version of 1 hopReason which does not use reasoning rule for construction, meaning that $V_{\text {in }}$ and $V_{\text {out }}$ are empty sets. All construction algorithms are executed 10 times on each instance. For the sake of fairness, heap is adopted by all competitor algorithms to speed up the construction.

In Tables 8 and $9,|V|$ and $|E|$ are reported to indicate the size of instances, and the size of $V_{\text {in }}^{G}$ and $V_{\text {out }}^{G}$ are also listed. The best solutions found by each construction method, 


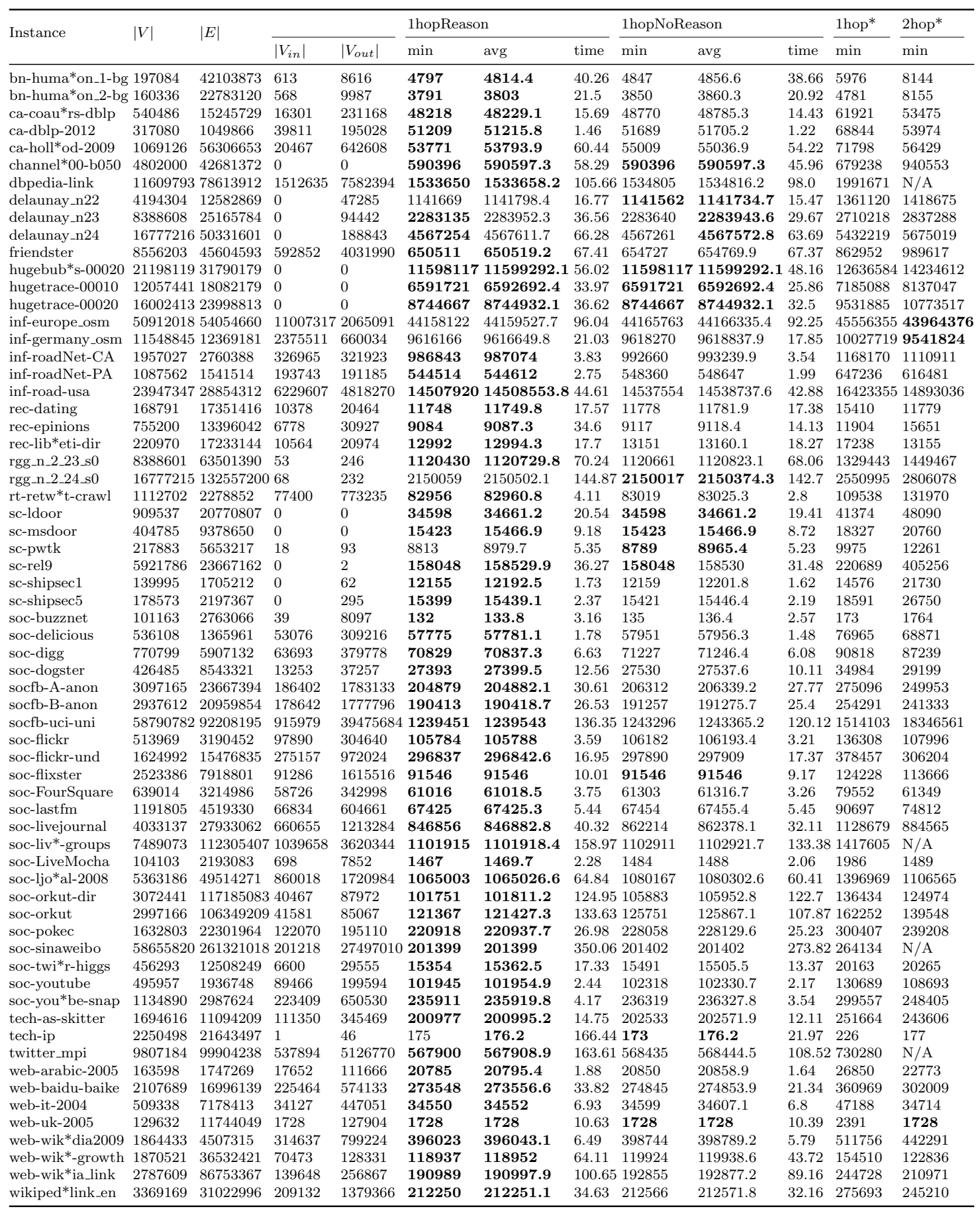

Table 9: Results of 1hopReason, 1hopNoReason, 1hop*, 2hop* on NDR benchmarks. 


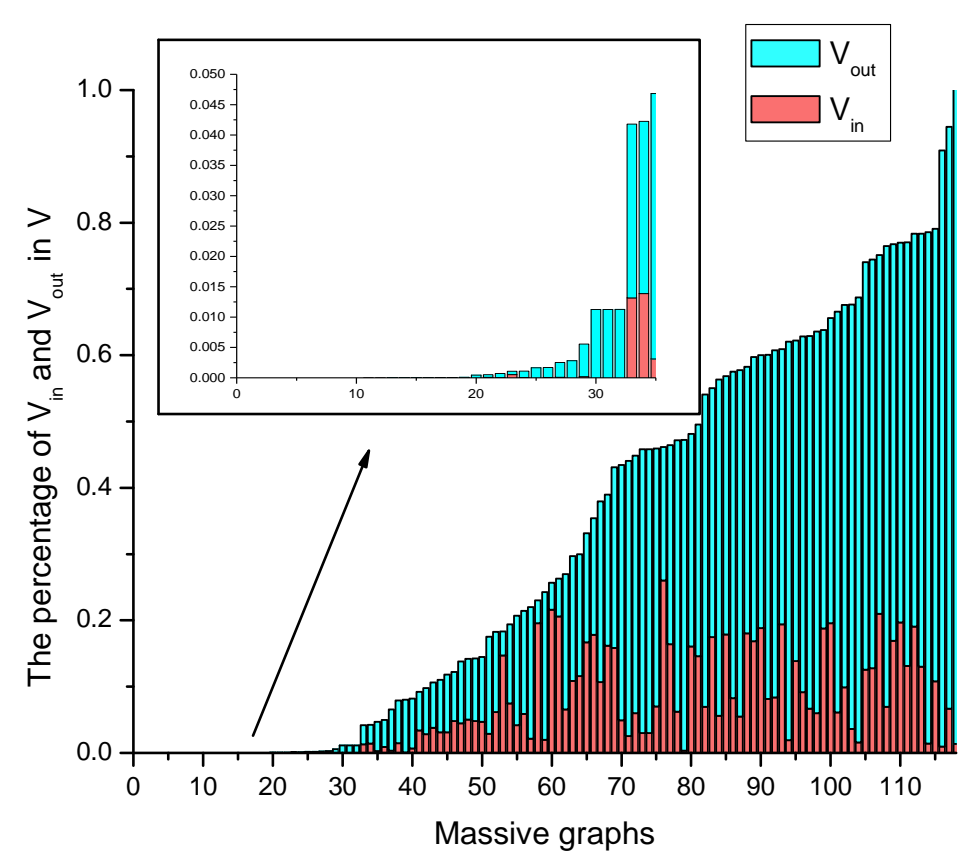

Figure 4: The percentage of the number of $V_{\text {in }}$ and $V_{\text {out }}$.

min, and the average size (avg) of the solutions found by 1hopReason and 1hopNoReason over 10 runs are reported. We also report the average time (in second) of 1hopReason and 1hopNoReason, time, to show the slight time overhead brought by calculating $\left|V_{i n}^{G}\right|$ and $\left|V_{\text {out }}^{G}\right|$. We report the detailed results on DIMACS10, NDR and SNAP here and the experimental results of all massive graphs are summarized in Table 10.

Moreover, we report the percentage of $V_{\text {in }}$ and $V_{\text {out }}$ in $V$ on 118 instances in Figure 4.

The results are summarized as follows.

- 1hopReason and 1hopNoReason can find better solution than 1hop* and 2hop* on almost all instances, indicating the effectiveness of our greedy construction method for massive graph.

- 1hopReason shows an overwhelming advantage over 1hopNoReason, confirming the effectiveness of the reasoning rule. The size of $V_{\text {in }}$ and $V_{\text {out }}$ and the comparison of time between 1 hopReason and 1 hopNoReason indicate that a considerable number of fixed vertices can be calculated within a short time for most instances. We observe that the reasoning method does not necessarily work if it meets some instances where only few vertices can be fixed.

- The Figure 4 indicates that the fixed vertices account for a large portion of all the vertices, thus significantly affecting the local search. 


\begin{tabular}{|c|c|c|c|c|c|c|c|c|c|c|}
\hline \multirow{2}{*}{ Benchmark } & \multicolumn{3}{|c|}{ 1hopReason } & \multicolumn{3}{|c|}{ 1hopNoReason } & \multicolumn{2}{|l|}{ 1hop* } & \multicolumn{2}{|l|}{2 hop* $^{*}$} \\
\hline & $\# \min$ & \#avg & time & $\# \min$ & \#avg & time & $\# \min$ & \#avg & $\# \min$ & \#avg \\
\hline DIMACS10(22) & 22 & 22 & 3.45 & 1 & 1 & 2.44 & 0 & 0 & 0 & 0 \\
\hline $\operatorname{SNAP}(31)$ & 22 & 24 & 24.73 & 11 & 9 & 23.14 & 13 & 14 & 2 & 2 \\
\hline $\operatorname{NRD}(65)$ & 59 & 58 & 46.24 & 13 & 14 & 39.01 & 0 & 0 & 3 & 3 \\
\hline
\end{tabular}

Table 10: Summarized results of 1hopReason, 1hopNoReason, 1hop*, 2hop* on DIMACS10, SNAP and NDR benchmarks.

\begin{tabular}{lllll}
\hline Benchmark & avg $(|V|)$ & avg $(|E|)$ & IoU_Pick & IoU_TBC \\
\hline DIMACS10 & 473156.09 & 2099769.55 & 0.007 & 0.703 \\
SNAP & 2258488.06 & 22850623.58 & 0.005 & 0.489 \\
NDR & 6394792.03 & 32477015.62 & 0.012 & 0.433 \\
\hline
\end{tabular}

Table 11: Different IoU on massive benchmarks. avg $(|V|)$ and avg $(|E|)$ denote the average number of vertices and edges in each benchmark.

\subsection{Further Analysis to HDC+ Effectiveness}

As stated in Section 4.3, the intuition of HDC+ is to take profit from respective advantages of TBC and SUB. Experimental results show that HDC+ is quite effective for massive graphs. We observe that the vertices picked in SUB and TBC significantly differ from each other, which provides an explanation to the success of HDC+ mechanism. In this subsection, extensive experiments are conducted to statistically confirm our observation. FastCDS is executed once and the cutoff time is set to 10000 seconds for the HDC+ analysis.

Given two vertex sets $S_{1}$ and $S_{2}, \frac{S_{1} \cap S_{2}}{S_{1} \cup S_{2}}$ is denoted as intersection-over-union (IoU) (Rezatofighi et al., 2019), which is adopted in Table 11 to analyse the performance of HDC+. Since TBC and SUB have same search space of adding vertices, we only consider the removing vertex set.

For IoU_Pick, $S_{1}$ is the picked vertex set of one SUB process, and $S_{2}$ is the picked vertex set of its following TBC process. Since the picked vertex set of TBC is much larger than that of SUB, for the sake of comparison, the former set only consists of top $k_{1}$ vertices selected in TBC with the the greatest $p i c k_{f}^{7}$ value, where $k_{1}$ is the number of vertices chosen in SUB. For IoU_TBC, $S_{1}$ and $S_{2}$ are the removed vertex sets for two consecutive TBC processes truncated by one SUB process. In Table 11, IoU_Pick and IoU_TBC are exhibited in the form of mean value among the benchmark. Observed from the results, we can obtain that:

- IoU_Pick indicates that vertices picked in SUB significantly differ from those in the following TBC. For further analysis, vertices picked in SUB and TBC are sorted in descending order according to pick $_{f}$. The top 20 vertices picked by SUB, which are the most frequently chosen ones, rank on average 34110.65 among those vertices picked by TBC. The above result ignores vertices not picked in TBC. For the top 20 vertices

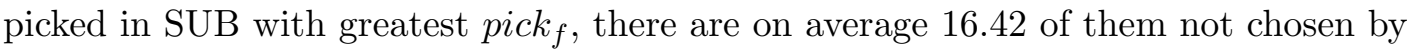

7. $\operatorname{pick}_{f}(v)$ is used to denote the frequency that vertex $v$ is chosen when using one connectivity method. 
the following TBC. The results show that SUB and TBC focus on different vertices, resulting in diversifying the search space.

- IoU_TBC indicates that the vertices chosen in two consecutive TBC processes truncated by SUB differ from each other. Moreover, IoU_TBC decreases as the size of input graph grows. The results show that HDC + mechanism significantly diversifies the search space, particularly when solving massive graphs.

We further analysed the steps of SUB and TBC. The experiment is carried out on DIMACS10, SNAP and NDR with the cutoff time of 3600 seconds. The average execution steps of SUB and TBC are 19652.45 and 43576123.07, respectively. The average number of switching between the SUB and TBC is 91.88. The average run-time for SUB and TBC are 204.39 seconds and 3382.34 seconds, respectively. TBC takes up the main proportion of time. SUB accounts for $0.045 \%$ steps, while it takes $5.700 \%$ time, which confirms the efficiency of TBC heuristic.

\subsection{Critical Difference Analysis}

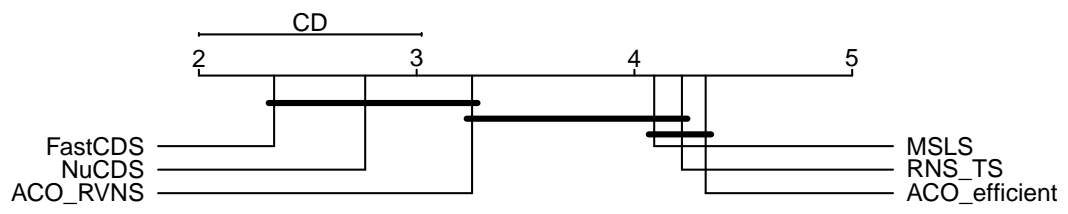

(a) classic benchmarks

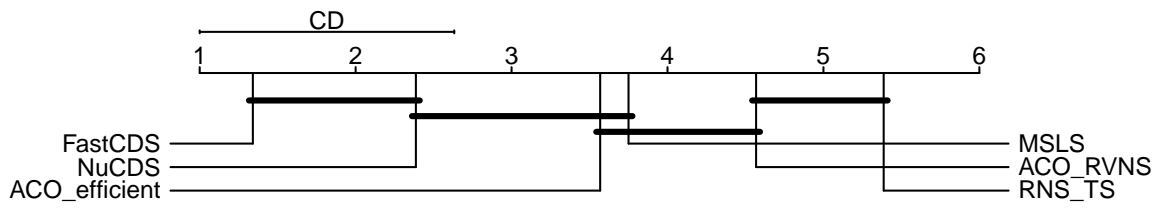

(b) DIMACS10

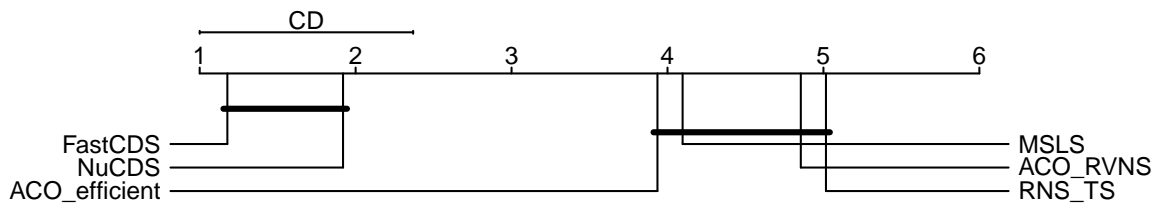

(c) SNAP

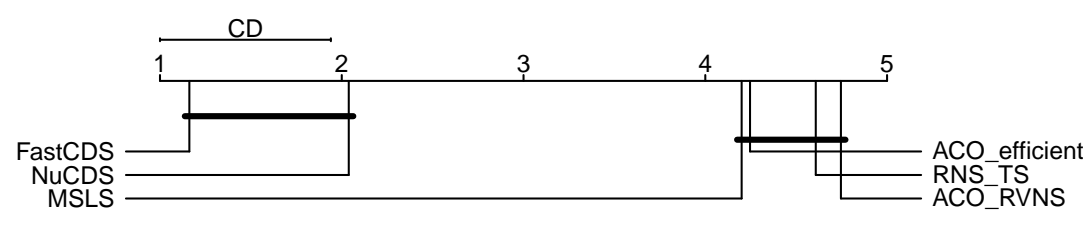

(d) NDR

Figure 5: Critical difference plots about FastCDS, NuCDS, MSLS, ACO-efficient, ACORVNS, RNS-TS on each benchmark. 
In this subsection, we evaluate the statistical differences between the considered algorithms on each benchmark. First, Friedman Test (Friedman, 1937) was conducted with a null-hypothesis that all the algorithms are equivalent in terms of the performance. After the null-hypothesis was rejected, all pairwise comparisons were performed using Nemenyi post-hoc test. Finally, the results are shown in Figure 5 in the form of critical difference diagram (Garcia \& Herrera, 2008). Note that we use a package of $\mathrm{R}$ called scmamp (Calvo \& Santafé, 2016), which can be found at https://github.com/b0rxa/scmamp. The top line in the diagram is the axis on which it plot the average ranks of algorithms, and the lower the ranks, the better the algorithm. The critical difference is shown above each sub-figure and the algorithms that are not significantly different with significant level of 0.05 are connected. From Figure 5, FastCDS outperforms other competitors on each benchmark.

\section{Conclusion}

We proposed three new algorithmic components for MCDS problem, namely the reasoning based construction, the hybrid dynamic connectivity maintenance heuristic and the two-level vertex selection heuristic. An efficient local search algorithm named FastCDS was developed based on the three components. Extensive experiments were conducted to evaluate the performance of FastCDS. Experiments showed that FastCDS could outperform other state-of-the-art algorithms on almost all the instances, and confirmed the effectiveness of three components.

For future work, we found that our proposed framework designed for solving MCDS problem is not suitable for the weighted version through simple adjustments. Thus, we plan to further study the minimum weight connected dominating set problem, which is a generalization of MCDS problem. Inspired by the success of $\mathrm{HDC}+$, we plan to apply it to other combinatorial optimization problem with connectivity constraint on massive graphs. Additionally, we would like to design a new algorithm for dynamic graphs which support incremental solving, rather than static graphs for the adaption of network applications in real time, such as the application of finding backbone in ever-changing wireless networks.

\section{Acknowledgments}

Shaowei Cai and Yiyuan Wang are corresponding authors. We would like to thank the anonymous referees for their helpful comments. This work was supported by Beijing Academy of Artificial Intelligence (BAAI), Youth Innovation Promotion Association, Chinese Academy of Sciences [No. 2017150], NSFC Grant 61806050, and the Fundamental Research Funds for the Central Universities 2412020FZ030.

\section{References}

Al-Karaki, J. N., \& Kamal, A. E. (2008). Efficient virtual-backbone routing in mobile ad hoc networks. Computer Networks, 52(2), 327-350.

Binkele-Raible, D., \& Fernau, H. (2012). An exact exponential-time algorithm for the directed maximum leaf spanning tree problem. Journal of Discrete Algorithms, 15, 
$43-55$.

Bouamama, S., Blum, C., \& Fages, J.-G. (2019). An algorithm based on ant colony optimization for the minimum connected dominating set problem. Applied Soft Computing, $80,672-686$.

Cai, S. (2015). Balance between complexity and quality: Local search for minimum vertex cover in massive graphs. In Proceedings of the Twenty-Fourth International Joint Conference on Artificial Intelligence, IJCAI 2015, Buenos Aires, Argentina, July 2531, 2015, pp. 747-753. AAAI Press.

Cai, S., Hou, W., Wang, Y., Luo, C., \& Lin, Q. (2020). Two-goal local search and inference rules for minimum dominating set. In Proceedings of the Twenty-Ninth International Joint Conference on Artificial Intelligence, IJCAI 2020, pp. 1467-1473. ijcai.org.

Cai, S., \& Su, K. (2011). Local search with configuration checking for SAT. In IEEE 23rd International Conference on Tools with Artificial Intelligence, ICTAI 2011, Boca Raton, FL, USA, November 7-9, 2011, pp. 59-66. IEEE Computer Society.

Cai, S., \& Su, K. (2013). Local search for boolean satisfiability with configuration checking and subscore. Artificial Intelligence, 204, 75-98.

Calvo, B., \& Santafé, G. (2016). scmamp: Statistical comparison of multiple algorithms in multiple problems. $R$ J., $8(1), 248$.

Chen, B., Jamieson, K., Balakrishnan, H., \& Morris, R. (2002). Span: An energy-efficient coordination algorithm for topology maintenance in ad hoc wireless networks. Wireless networks, 8(5), 481-494.

Chen, S., Ljubić, I., \& Raghavan, S. (2010). The regenerator location problem. Networks: An International Journal, 55(3), 205-220.

Cheng, X., Ding, M., Du, D. H., \& Jia, X. (2006). Virtual backbone construction in multihop ad hoc wireless networks. Wireless Communications and Mobile Computing, 6(2), $183-190$.

Cheng, X., Huang, X., Li, D., Wu, W., \& Du, D.-Z. (2003). A polynomial-time approximation scheme for the minimum-connected dominating set in ad hoc wireless networks. Networks: An International Journal, 42(4), 202-208.

Chinnasamy, A., Sivakumar, B., Selvakumari, P., \& Suresh, A. (2019). Minimum connected dominating set based $\mathrm{rsu}$ allocation for smartcloud vehicles in vanet. Cluster Computing, 22(5), 12795-12804.

Dagdeviren, Z. A., Aydin, D., \& Cinsdikici, M. (2017). Two population-based optimization algorithms for minimum weight connected dominating set problem. Applied Soft Computing, 59, 644-658.

Deb, B., Bhatnagar, S., \& Nath, B. (2003). Multi-resolution state retrieval in sensor networks. In Proceedings of the First IEEE International Workshop on Sensor Network Protocols and Applications, 2003., pp. 19-29. IEEE.

Erdem, E., Lin, F., \& Schaub, T. (Eds.). (2009). Proceedings of 10th International Conference on Logic Programming and Nonmonotonic Reasoning, Vol. 5753 of Lecture Notes in Computer Science. 
Fan, N., \& Watson, J.-P. (2012). Solving the connected dominating set problem and power dominating set problem by integer programming. In International conference on combinatorial optimization and applications, pp. 371-383.

Fernau, H., Kneis, J., Kratsch, D., Langer, A., Liedloff, M., Raible, D., \& Rossmanith, P. (2011). An exact algorithm for the maximum leaf spanning tree problem. Theoretical Computer Science, 412(45), 6290-6302.

Fomin, F. V., Grandoni, F., \& Kratsch, D. (2008). Solving connected dominating set faster than 2 n. Algorithmica, 52(2), 153-166.

Friedman, M. (1937). The use of ranks to avoid the assumption of normality implicit in the analysis of variance. Journal of the american statistical association, 32(200), 675-701.

Garcia, S., \& Herrera, F. (2008). An extension on" statistical comparisons of classifiers over multiple data sets" for all pairwise comparisons.. Journal of machine learning research, 9(12).

Gendron, B., Lucena, A., da Cunha, A. S., \& Simonetti, L. (2014). Benders decomposition, branch-and-cut, and hybrid algorithms for the minimum connected dominating set problem. INFORMS Journal on Computing, 26(4), 645-657.

Glover, F., \& Laguna, M. (1998). Tabu search. In Handbook of combinatorial optimization, pp. 2093-2229. Springer.

Hedar, A.-R., \& Ismail, R. (2012). Simulated annealing with stochastic local search for minimum dominating set problem. International Journal of Machine Learning and Cybernetics, 3(2), 97-109.

Hedar, A.-R., Ismail, R., El-Sayed, G. A., \& Khayyat, K. M. J. (2019). Two meta-heuristics designed to solve the minimum connected dominating set problem for wireless networks design and management. Journal of Network and Systems Management, 27(3), 647-687.

Hopcroft, J., \& Tarjan, R. (1973). Algorithm 447: efficient algorithms for graph manipulation. Communications of the ACM, 16(6), 372-378.

Jovanovic, R., \& Tuba, M. (2013). Ant colony optimization algorithm with pheromone correction strategy for the minimum connected dominating set problem.. Comput. Sci. Inf. Syst., 10(1), 133-149.

Kann, V. (1992). On the approximability of NP-complete optimization problems. Ph.D. thesis, Royal Institute of Technology Stockholm.

Khuller, S., \& Yang, S. (2019). Revisiting connected dominating sets: An almost optimal local information algorithm. Algorithmica, 81 (6), 2592-2605.

Li, B., Zhang, X., Cai, S., Lin, J., Wang, Y., \& Blum, C. (2020a). Nucds: An efficient local search algorithm for minimum connected dominating set. In Proceedings of the Twenty-Ninth International Joint Conference on Artificial Intelligence, IJCAI-20, pp. $1503-1510$.

Li, J., Wen, X., Wu, M., Liu, F., \& Li, S. (2020b). Identification of key nodes and vital edges in aviation network based on minimum connected dominating set. Physica A: Statistical Mechanics and its Applications, 541, 123340. 
Li, R., Hu, S., Gao, J., Zhou, Y., Wang, Y., \& Yin, M. (2017). Grasp for connected dominating set problems. Neural Computing and Applications, 28(1), 1059-1067.

Li, R., Hu, S., Liu, H., Li, R., Ouyang, D., \& Yin, M. (2019). Multi-start local search algorithm for the minimum connected dominating set problems. Mathematics, 7(12), 1173.

Lin, J., Cai, S., Luo, C., \& Su, K. (2017). A reduction based method for coloring very large graphs.. In $I J C A I$, pp. 517-523.

Lucena, A., Maculan, N., \& Simonetti, L. (2010). Reformulations and solution algorithms for the maximum leaf spanning tree problem. Computational Management Science, 7(3), 289-311.

Milenković, T., Memišević, V., Bonato, A., \& Pržulj, N. (2011). Dominating biological networks. PloS one, 6(8), e23016.

Misra, R., \& Mandal, C. (2009). Minimum connected dominating set using a collaborative cover heuristic for ad hoc sensor networks. IEEE Transactions on parallel and distributed systems, $21(3), 292-302$.

Morgan, M., \& Grout, V. (2007). Metaheuristics for wireless network optimisation. In $A I C T$, pp. $15-15$.

Ni, S.-Y., Tseng, Y.-C., Chen, Y.-S., \& Sheu, J.-P. (1999). The broadcast storm problem in a mobile ad hoc network. In Proceedings of the 5th annual ACM/IEEE international conference on Mobile computing and networking, pp. 151-162. ACM.

Rezatofighi, H., Tsoi, N., Gwak, J., Sadeghian, A., Reid, I., \& Savarese, S. (2019). Generalized intersection over union: A metric and a loss for bounding box regression. In Proceedings of the IEEE Conference on Computer Vision and Pattern Recognition, pp. 658-666.

Rossi, R. A., \& Ahmed, N. K. (2015). The network data repository with interactive graph analytics and visualization. In Proceedings of the Twenty-Ninth AAAI Conference on Artificial Intelligence, pp. 4292-4293.

Ruan, L., Du, H., Jia, X., Wu, W., Li, Y., \& Ko, K.-I. (2004). A greedy approximation for minimum connected dominating sets. Theoretical Computer Science, 329(1-3), $325-330$.

Sen, A., Murthy, S., \& Bandyopadhyay, S. (2008). On sparse placement of regenerator nodes in translucent optical network. In IEEE GLOBECOM 2008-2008 IEEE Global Telecommunications Conference, pp. 1-6. IEEE.

Simonetti, L., Da Cunha, A. S., \& Lucena, A. (2011). The minimum connected dominating set problem: Formulation, valid inequalities and a branch-and-cut algorithm. In International Conference on Network Optimization, pp. 162-169. Springer.

Solis-Oba, R., Bonsma, P., \& Lowski, S. (2017). A 2-approximation algorithm for finding a spanning tree with maximum number of leaves. Algorithmica, $77(2), 374-388$.

Wang, Y., Cai, S., \& Yin, M. (2017). Local search for minimum weight dominating set with two-level configuration checking and frequency based scoring function. Journal of Artificial Intelligence Research, 58, 267-295. 
Wu, J., \& Dai, F. (2003). Broadcasting in ad hoc networks based on self-pruning. International Journal of Foundations of Computer Science, 14(02), 201-221.

Wu, X., Lü, Z., \& Galinier, P. (2017). Restricted swap-based neighborhood search for the minimum connected dominating set problem. Networks, 69(2), 222-236.

Yu, J., Wang, N., Wang, G., \& Yu, D. (2013). Connected dominating sets in wireless ad hoc and sensor networks-a comprehensive survey. Computer Communications, 36(2), $121-134$. 